\title{
Artículos
}

\section{Una propuesta metodológica para el análisis intertemporal de la conmutación laboral: el caso de la Patagonia chilena}

\section{A methodological proposal for an intertemporal analysis of labour commutation: The case of Chilean Patagonia}

\author{
Claudio Mancilla* \\ Sergio Soza-Amigo** \\ Luz María Ferrada***
}

\section{Resumen}

El objetivo del presente trabajo es aplicar una propuesta metodológica para identificar las características de la conmutación laboral entre dos periodos en la Patagonia chilena, con datos agrupados. Se analizan los centros nodales principales, el origen, los sectores productivos y el nivel de estudios de los trabajadores. Se utilizó la Encuesta Suplementaria de Ingresos de los años 2011 y 2016. Se propone el cálculo de un índice y se aplica una prueba no paramétrica. Los principales resultados indican que los centros nodales están poco conectados entre ellos y, contrario a lo esperado, los trabajadores con bajo nivel de estudios explican principalmente la conmutación de más larga distancia.

* Universidad de Los Lagos. Departamento de Ciencias Administrativas y Económicas. Dirección: Fuschlocher 1305, Osorno, Los Lagos, Chile. Correo: claudio.mancilla@ulagos.cl ORCID: https://orcid.org/0000-0003-2864-2112

** Universidad Austral de Chile, Instituto de Gestión e Industria. Dirección: Los Pinos s/n, Balneario Pelluco, Puerto Montt, Chile. Correo: sergio.soza@uach.cl ORCID: https:// orcid.org/0000-0002-8256-6782

*** Universidad de Los Lagos, Departamento de Ciencias Administrativas y Económicas. Osorno, Chile. Correo electrónico: 1ferrada@ulagos.cl ORCID: https://orcid.org/00000002-0481-7670

Nota de los autores: Este trabajo está enmarcado dentro del proyecto de investigación "La vocación productiva, el nivel educativo y la funcionalidad del centro emisor y receptor como factores explicativos de la conmutación inter e intrarregional en la Región de los Andes Australes de la Patagonia Chilena", financiado por Conicyt (proyecto Fondecyt núm. 1160196), por lo que los autores agradecen dichos aportes. 
Palabras clave: índice de similitud temporal; conmutación laboral; Patagonia chilena; sectores productivos; nivel educacional.

\begin{abstract}
The objective of this paper is to apply a methodological proposal to identify the main characteristics of labor commutation in Chilean Patagonia, with grouped data from two periods. The main nodal centers, the origin, the productive sectors, and the educational level of the workers were analyzed. Data was derived from the Supplementary Income Survey for the years 2011 and 2016. Through applying an index and a non-parametric test, results indicate there is little connection between the main nodal centers and the Patagonian regions and, contrary to expectations, the lower education levels of some workers explain longer distance commutation.
\end{abstract}

Keywords: temporal similarity index; commutation; Chilean Patagonia; productive sectors; educational level.

\title{
Introducción
}

La conmutación en la Patagonia chilena ha sido comparativamente poco estudiada, por lo que las características globales del fenómeno no son claras en este territorio. La existencia de pocos estudios está explicada, entre otras causas, por la escasez de información y porque las bases de datos existentes son poco representativas para este espacio geográfico, dificultándose así el proceso de inferencia estadística. Dado lo anterior, este trabajo propone un diseño metodológico para analizar el fenómeno de la conmutación en dicho territorio, que además puede ser aplicado de forma simple para el análisis de otros espacios con similares características y restricciones de información.

La conmutación laboral es un fenómeno importante para el análisis económico y del espacio geográfico ya que puede generar efectos importantes en las economías locales, es decir, tiene impacto tanto en los territorios de origen como en los de destino (Aroca y Atienza, 2007; Muñoz, 2012). En el caso particular de Chile, la conmutación interregional es un fenómeno creciente que sobrepasa a la migración interregional (Aroca y Atienza, 2007).

Los estudios realizados indican que la conmutación laboral ha sido abordada mayormente desde una perspectiva de conmutación de larga distancia o en el interior de zonas metropolitanas (Canales y Canales, 2016), más que como una conmutación entre regiones más cercanas, lo que pone de manifiesto la necesidad de comprender mejor el fenómeno incorporando también una perspectiva intermedia. 
Pino, Concha y Fuentes (2016) indican que la conmutación laboral en Chile creció entre 2011 y 2014 en $23 \%$. Considerando los cálculos propios de los autores de este trabajo, y en conformidad con la Encuesta Suplementaria de Ingresos (ESI) del Instituto Nacional de Estadísticas de Chile (INE), se observa en las regiones (primera división política administrativa) de la Patagonia que el total de ocupados en los centros nodales principales (CNP) aumentó $10.2 \%$ entre los años 2011 y 2016 , mientras que los conmutantes laborales hacia los CNP crecieron en $34 \%$.

Sumado a lo anterior, se observa que para el caso de la Patagonia existen limitantes, tales como la información representativa del territorio. Dado esto último, en este trabajo se propone un diseño metodológico para analizar el fenómeno de la conmutación en la Patagonia, que puede ser aplicado de forma simple para el análisis de otros espacios con similares características y restricciones de información.

Como una forma de enmarcar el trabajo que se desarrollará, la conmutación laboral será entendida aquí como un fenómeno que se refiere al hecho de que la ciudad donde vive una persona es diferente a la ciudad donde trabaja. En este último caso, se hará referencia a los centros nodales (ciudades o comunas) principales en la Patagonia chilena.

En resumen, se propone un procedimiento sencillo de seguir, pero que contribuye a generar preguntas y aproximaciones para analizar regiones similares a la Patagonia chilena, de las que se posea poca información para estudiar la conmutación, con características tales como las de ser zonas relativamente aisladas o remotas, pero pertenecientes a macrozonas, con un importante énfasis en la explotación de recursos naturales y agrícolas, salarios relativamente más bajos a los de las zonas metropolitanas y con baja disponibilidad de mano de obra, tanto en cantidad como en cualificación. El análisis se centra en los conmutantes que llegan a los cinco centros nodales principales de la Patagonia (ordenados de norte a sur): Valdivia, Osorno, Puerto Montt, Coyhaique y Punta Arenas.

Específicamente, en lo que sigue, se utiliza una estrategia de agrupación de datos; posteriormente se aplica una prueba no paramétrica para determinar la existencia de una relación o independencia entre variables, y finalmente se propone el cálculo de un índice de similitud temporal para analizar el cambio de la distribución de los conmutantes laborales entre los años 2011 y 2016.

De esta manera, este trabajo tiene como objetivo aplicar la metodología propuesta para identificar las características de la conmutación laboral entre dos periodos en la Patagonia chilena, con datos agrupados. Se generan proposiciones de acuerdo a diversos autores, por ejemplo, el hecho de que 
se esperaría que existiera una relación inversa entre la distancia y el flujo de conmutantes, así como una relación entre el nivel de estudios de los trabajadores, los sectores productivos y el tipo de conmutación que se realiza. Además, se dimensiona la fricción-distancia con datos agrupados dada la inexistencia de microdatos que sean representativos, con el fin de potenciar el análisis de lo propuesto.

\section{Antecedentes y aspectos teóricos-empíricos}

La Patagonia es parte de América del Sur y se divide en la occidental, que pertenece a Chile, y la oriental, que corresponde a Argentina. Para el caso chileno, es posible encontrar autores que fijan el límite norte en distintos puntos. El más septentrional es situado por los historiadores Enrique Campos Menéndez y Raúl Rey Balmaceda en el norte de la ciudad de Valdivia (Said, 2012), a partir del paralelo 3949’ (véase el Mapa 1).

De acuerdo a la división político-administrativa de Chile, es posible definir cuatro regiones dentro de la Patagonia, denominadas, de norte a sur: Los Ríos, Los Lagos, Aysén del General Carlos Ibáñez del Campo, y la región de Magallanes y Antártica Chilena. Aunque hay decenas de ciudades y pueblos, en el año 2011 eran cinco los centros urbanos que concentraban casi el 49.5\% de su población, mientras que en 2016 concentraban el 50.1\%: Valdivia, Osorno, Puerto Montt, Coyhaique y Punta Arenas (cálculos propios conforme a datos del Instituto Nacional de Estadísticas de Chile, INE) (véase el Mapa 1). A estas ciudades principales se les denominará centros nodales principales (CNP), por cuanto, de acuerdo a Soza-Amigo (2016b), estas urbes tienen la capacidad de interactuar estructural y funcionalmente a partir de indicadores económicos y demográficos en función de los servicios que entregan a la comunidad.

Estos centros nodales poseen algunas diferencias en su origen y desarrollo. Valdivia y Osorno son las ciudades más antiguas, fundadas en el siglo XVI, y experimentaron junto a Puerto Montt una importante inmigración de origen alemán entre finales del siglo XIX y principios del XX. Además, por su cercanía geográfica, podrían eventualmente tener una mayor vinculación. Por otra parte, Punta Arenas, en el mismo periodo, dada su ubicación estratégica en el Estrecho de Magallanes, también tuvo inmigración europea, aunque ésta fue más diversa. Por el lado de Coyhaique, que tiene sus inicios en el siglo XIX, su desarrollo estuvo vinculado a una colonización por parte del Estado de Chile. 


\section{Mapa 1}

Chile, división político-administrativa: centros nodales principales de la Patagonia chilena

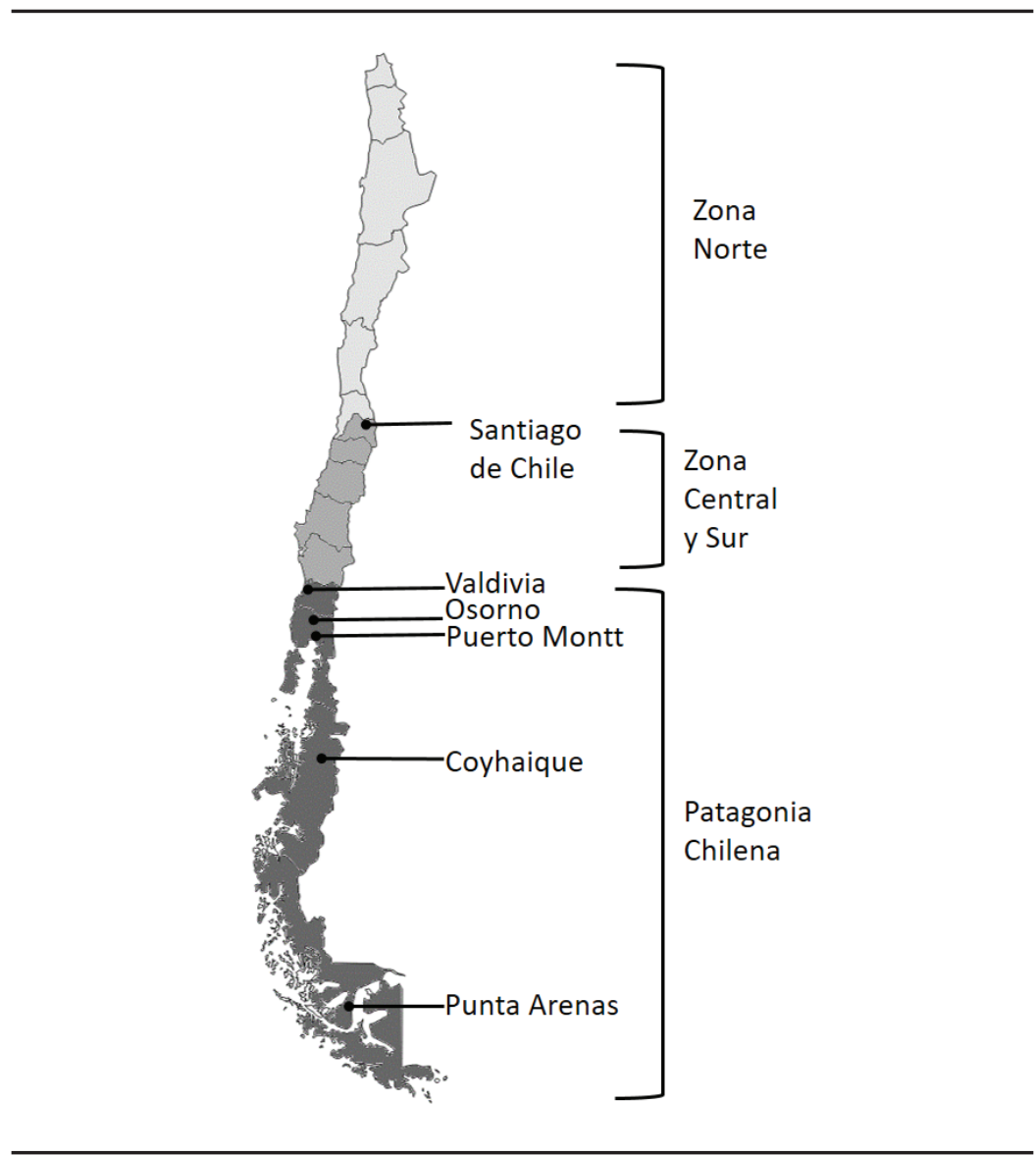

Fuente: Elaboración propia con base en Said, 2012.

\section{La conmutación y las regiones policéntricas}

De acuerdo a Yáñez y Acevedo (2010) existen dos tipos de trabajos que han abordado el espacio geográfico y el mercado laboral: los que buscan explicar la localización de la actividad económica, y los que abordan la hipótesis de mismatch espacial, que es la falta de correspondencia entre el lugar de 
residencia y el del empleo, y que correspondería al fenómeno de la conmutación que se aborda en este trabajo. Algunos aspectos que Yáñez y Acevedo señalan de los trabajos que han tratado el mismatch espacial son aquellos que explican la no correspondencia debido a la carencia de información de empleos en el lugar de residencia, o a que el empleo crece a una tasa insuficiente, y la importancia de los vínculos sociales de las personas para encontrar trabajo. A pesar de lo anterior, desde el punto de vista teórico, no existe un modelo explícito y único que aborde el fenómeno de la conmutación laboral interregional tal como se plantea en esta investigación.

Sin embargo, desde el modelo de Alonso, del año 1960, se puede interpretar que los costos de transporte explican en gran medida la conmutación, ya que influyen en la decisión de los individuos para escoger los lugares de vivienda y trabajo. Entre las variables que explican esta decisión se encuentra el costo de la tierra, que en la actualidad haría referencia directa al costo de compra o alquiler de la vivienda. Sin embargo, dado el avance tecnológico y los costos y tiempos del transporte, se puede desprender desde el modelo que los individuos, una vez localizados en su residencia y frente al escenario de no tener o perder el empleo, decidirán conmutar si los ingresos compensan los costos.

Autores como Aroca y Atienza (2007) indican que la conmutación interregional ha sido, comparativamente, menos estudiada, especialmente en las zonas extremas (norte y sur de Chile). Además, la conmutación en el país probablemente está explicada por una baja en los costos de transporte; asimismo, los centros urbanos o regiones que tienen más población tienden a tener mayores niveles de precios de viviendas. Así, el costo de la vida, expresado como el costo de la vivienda, estaría incentivando la conmutación en el país (Aroca y Atienza, 2008). Ábalos y Paredes (2014, p. 104) indican que la conmutación tiene asociada la interacción de unidades espaciales que hacen que el mercado del trabajo sea consecuencia de la existencia de estos territorios, y si bien estas unidades en apariencia pueden ser independientes, podrían estar funcionando como una macrounidad espacial.

No obstante, existen estudios internacionales que abordan la conmutación y que buscan comprender el fenómeno a una escala intermedia, como es la conmutación intrarregional e interregional, y no sólo de larga distancia. Algunos de estos autores son: Roberts (1998) en Escocia, Sandow (2008) en áreas poco pobladas en Suecia, Timmermans y Boschma (2014) en Dinamarca, y más recientemente Bergantino y Madio (2019) realizan un análisis para Reino Unido entre los años 2004 y 2014.

Canales y Canales (2016) describen que la conmutación de larga distancia, para el caso de Chile, se concentra en el norte y sur del país, mientras 
que la conmutación intrarregional, que se moviliza dentro de cada región político-administrativa, se concentra en la zona central del país. Esto develaría que existen patrones de movilidad diferentes que podrían ser extensibles a otras macrozonas.

Parr (2008) señala que existe una tendencia a tratar a las ciudades como regiones y a las regiones como ciudades, por lo que suele ser difícil aislar los conceptos, lo que al final resulta confuso. Esta situación se suele dar cuando se habla de grandes centros metropolitanos y se hace referencia a una región, cuando en realidad se quiere hablar de una red de ciudades alrededor de una ciudad principal. Dado lo anterior, parece ser más apropiado hacer mención de una región policéntrica, es decir, un territorio con varios centros urbanos.

Con base en lo expuesto, la Patagonia chilena puede ser conceptualizada como una unidad espacial policéntrica, dados los cinco centros nodales principales identificados. Sin embargo, también es necesario reflexionar sobre la ubicación de estos centros. En el Mapa 1 se observa que tres de ellos (Valdivia, Osorno y Puerto Montt) están comparativamente más cerca entre ellos y situados al norte, mientras que al sur están Coyhaique y Punta Arenas, que no sólo están más aislados entre sí, sino que también están mucho más distantes de los primeros centros nodales. Así, se puede entender que la Patagonia está dividida en al menos dos grandes zonas: la norte y la sur.

Ferrada, Soza-Amigo y Mancilla (2019) identifican estas diferencias geográficas e indican que las distancias de conmutación son menores en el norte en comparación con las que ocurren en la parte sur, lo que podría develar patrones de conmutación diferentes incluso en el interior de la Patagonia.

En relación con las regiones policéntricas, Parr (2004) discute acerca de este concepto. Específicamente, el autor se refiere a regiones urbanas policéntricas (PUR, Polycentric Urban Regions) en torno a las que, si bien existe acuerdo al considerarlas áreas subnacionales (regiones) con varios centros urbanos, el nivel máximo de separación es arbitrario. De lo anterior se desprende que el territorio policéntrico a analizar puede diferir según el criterio que se adopte.

Yinger (1992) indica que los territorios policéntricos tienden a tener una mayor conmutación de corta distancia y, dado que la Patagonia chilena es un territorio con varios centros, se puede esperar que la conmutación se asocie a lo indicado por este autor. Por otra parte, Parr (2004) señala que se espera que una región policéntrica tenga más vínculos en distintas dimensiones entre sus centros que cualquier otro territorio.

Conforme a lo anterior, considerando que los cinco centros urbanos más importantes que se analizan en este trabajo concentran para la zona de 
estudio casi el 50\% de la población, se espera que en la Patagonia chilena exista una importante conmutación dentro de una misma región político-administrativa (es decir, en torno al área más cercana). En este trabajo, a esta conmutación se le denominará conmutación intrarregional. En la misma línea, y en función de la distancia, se espera que el segundo flujo de personas provenga desde las otras regiones de la misma Patagonia, caso en el que hablaremos de conmutación interregional. En tercer lugar, la conmutación proveniente de regiones situadas al norte de la Patagonia será denominada conmutación extrarregional (conmutación más lejana). Lo anterior conduce a una primera hipótesis en este trabajo: existiría una relación inversa entre la distancia y el flujo de conmutantes hacia los centros nodales principales de la Patagonia chilena.

\section{Factores que inciden en la conmutación}

La conmutación suele estar asociada y explicada por factores como género, formación y sectores productivos (Ascencio y Pino, 2015). Otros aspectos como salarios, niveles de PIB per cápita, encadenamiento de sectores productivos y distancia (Soza-Amigo 2016a), también están relacionados al fenómeno. Asimismo, teorías clásicas de la migración (tratadas en Massey, Arango, Hugo, Kouaouci, Pellegrino y Taylor, 1993) vinculadas a una perspectiva neoclásica, indican que las diferencias en el mercado del trabajo entre los territorios, como los salarios y la disponibilidad de capital humano (cantidad y cualificación), pueden influir sobre las decisiones de migrar.

En el caso de la formación de las personas, Fujita y Krugman (2004) mencionan a la migración constante de individuos con mayores niveles de formación como elementos importantes para la difusión del conocimiento; así es posible visualizar que la conmutación de trabajadores cualificados es importante porque puede ser muy beneficiosa para la Patagonia chilena por cuanto estos individuos llevarían consigo conocimientos y experiencias cuyas externalidades positivas debieran reflejarse en los territorios de destino.

Aroca y Atienza (2007) indican que los conmutantes tienden a tener más escolaridad que los habitantes locales. Así, se esperaría que cuanto mayor sea el nivel de escolaridad de los individuos, éstos tendrían más flexibilidad laboral, así como también mayores ingresos que deberían compensar el costo de conmutar. De esta manera, la segunda hipótesis del presente trabajo para describir el fenómeno de conmutación en la Patagonia chilena indica que la conmutación de más larga distancia está asociada a niveles más altos de formación. 
La localización de los recursos naturales en Chile juega un rol importante para explicar el fenómeno de la conmutación; es así como la conmutación de larga distancia hacia el norte del país está explicada principalmente por la existencia del sector productivo de la minería del cobre (Ábalos y Paredes, 2014). A su vez, el tipo de encadenamiento entre los sectores, el PIB de los mismos y la estructura productiva también podrían ayudar a explicar la mayor conmutación (Soza-Amigo, 2016a; 2016b). Por su parte, Zhao, Lu y De Roo (2011) mencionan que el tipo de sector productivo (primario, secundario o terciario) en el que se desempeña un trabajador conmutante se relaciona con el tipo de conmutación (corta o larga distancia).

Los principales sectores o actividades económicas que dominan en los centros nodales principales de la Patagonia chilena, de acuerdo con Soza-Amigo, Mancilla y Ferrada (2018), son la ganadería, la industria no metálica, el transporte y el comercio. Por lo tanto, a diferencia de lo que ocurre en el norte de Chile, la Patagonia chilena es un territorio que no se caracteriza por una actividad primaria asociada a la minería, que paga en promedio salarios más altos (a excepción de Punta Arenas, con la extracción de gas natural que facilita la actividad del metanol). En la Patagonia, los mayores ingresos están en sectores productivos de tipo secundario (industria) y terciario (comercio y servicios); de esta manera, se puede esperar que exista un mayor flujo de conmutantes hacia este tipo de sectores. Así, la última hipótesis de este trabajo indica que la conmutación laboral en la Patagonia chilena está asociada principalmente a los sectores productivos terciarios y secundarios. Específicamente, al indicar sectores productivos secundarios, se hace referencia a aquéllos como la industria manufacturera, la construcción, la minería (aunque ésta tiene una mínima expresión en la Patagonia) y la generación y distribución de electricidad, gas y agua; mientras los sectores terciarios hacen referencia a los asociados al comercio y los servicios.

\section{Datos y propuesta metodológica}

La información para analizar la conmutación laboral en la Patagonia chilena proviene de la Encuesta Suplementaria de Ingresos (ESI) para dos años: 2011 y 2016. La información es generada por el Instituto Nacional de Estadísticas de Chile (INE), desde donde es posible identificar en qué centro nodal trabaja una persona, el sector productivo en el que se desempeña y el nivel de formación o estudios que posee.

La selección de los años permite comparar entre dos periodos, con una diferencia de cinco años, aunque en el futuro, al ser una encuesta anual, será 
posible hacer comparaciones intertemporales más amplias. A la fecha del presente trabajo, la información del año 2016 era la última disponible. Se debe indicar que, aunque era posible considerar la información de 2010 -la primera con la actual metodología de levantamiento de datos del INE-, ésta se excluyó del análisis porque en dicho año existió un importante terremoto en la zona de la Región del Bío-Bío, al norte de la Patagonia, lo que podría eventualmente haber afectado parte de la infraestructura y del mercado del trabajo.

La información utilizada tiene limitaciones en cuanto a su representatividad a nivel de ciudades, por lo que la estrategia en torno al tratamiento de los datos comprenderá el análisis de los resultados de forma agregada, utilizando los factores de expansión proporcionados por la base de datos. Así, dado que los resultados son representativos a nivel de regiones (primera división político administrativa), se analizará a los conmutantes desde la perspectiva de cómo se mueven desde las regiones hacia los centros nodales principales (la limitación de representatividad no permitiría, por ejemplo, realizar un análisis significativo de conmutación neta de cada uno de los centros nodales).

Como segundo paso, para probar las hipótesis anteriormente mostradas, se construirán tablas de contingencia. Para verificar si existe alguna asociación o independencia significativa entre las variables o las dimensiones de las tablas (centros nodales, tipo de conmutación, sectores productivos y/o nivel de formación), y para cada año, se aplicará la prueba no paramétrica de asociación o independencia Chi-2 (Ecuación 1). Esta prueba permite la comparación de dos atributos (variables) para determinar si existe una relación entre ellos. Si se rechaza la hipótesis de nula independencia, se podrán realizar análisis que consideren una asociación entre las categorías de las dos variables (Webster, 2000).

$$
\chi^{2}=\sum_{i=1}^{k} \frac{\left(O_{i}-E_{i}\right)^{2}}{E_{i}}
$$

donde: $k$ es el número de categorías o clases, $O_{i}$ es la frecuencia observada, $E_{i}$ es la frecuencia esperada si la hipótesis nula es correcta.

Para una mejor compresión y análisis, se construirán gráficos circulares para visualizar los flujos de conmutación y su distribución entre variables o dimensiones de análisis. Para lo anterior, se utiliza la herramienta desarrollada por Krzywinski, Schein, Birol, Connors, Gascoyne, Horsman, Jones y Marra (2009), que originalmente fue propuesta para investigaciones en el 
área de la genética. Uno de los primeros trabajos en utilizar estos gráficos para flujos migratorios es el de Abel y Sander (2014), quienes los introducen para exponer sus análisis de 196 países, y con ello exponer fácilmente los resultados y conclusiones.

Un tercer procedimiento consiste en determinar las diferencias en la distribución de conmutantes entre el año 2011 y el 2016, considerando las variables de centro nodal principal, sector productivo, tipo de conmutación (intra, inter y extrarregional) y nivel de formación. Ya que sólo se podrá establecer un cruce entre dos variables, a cada análisis cruzado o de tabla de contingencia se le denominará dimensión de análisis.

Al desarrollar un análisis de dos años, nos encontraremos frente a dos matrices, cada una de ellas con distintos valores y probablemente con diferente distribución relativa de los datos. Así, para dimensionar la existencia de una diferencia de distribución entre los años, se propone el cálculo de un indicador que es una adaptación del coeficiente de especialización (Lira y Quiroga, 2008) y el índice de Le Masne (1988). El primero permite conocer si la distribución relativa de la producción (o cualquier otra variable) de un territorio es similar a la de otro territorio (el utilizado como patrón de comparación); sin embargo, este indicador está pensado para comparar dos vectores columna o vectores fila (Ecuación 2) y su resultado va de cero a uno, donde cero indicaría completa similitud de la estructura productiva (o variable de análisis) entre dos territorios. Para realizar el cálculo, primero se hace necesario relativizar los valores a proporciones o porcentajes.

Por su parte, el indicador de Le Masne, utilizado por ejemplo en el trabajo de Soza-Amigo, Mancilla y Ferrada (2017) y que se presenta en la Ecuación 3, permite cotejar en un análisis económico de matriz insumo-producto los coeficientes técnicos de dos matrices. En dicho análisis se hace necesario corregir el tamaño de las economías para establecer la comparación. El resultado del indicador está entre 0 y 100; si el índice arroja un valor próximo a 100, mostrará una alta similitud en las estructuras que se cotejan, mientras que valores cercanos a cero señalarán lo contrario. Es decir, el indicador de Le Masne tiene una interpretación completamente distinta a la del primero de los indicadores mencionados.

Tomando como base lo anteriormente expuesto, se construye la Ecuación 4, que se utilizará para analizar el cambio intertemporal de la distribución de conmutantes, expresión a la que se le ha denominado índice de similitud intertemporal. En primer lugar, desde del índice de especialización (Ecuación 2) se adoptará la transformación del valor de cada una de las celdas de la matriz en valores relativos respecto de la sumatoria total de los valores de la matriz, es decir, se comparará la proporción o distribución 
porcentual de los conmutantes en una matriz; en segundo lugar, se adoptará la estrategia para comparar dos matrices y la interpretación del índice de Le Masne (Ecuación 2), donde un valor cercano a 100 indicaría alta similitud de la distribución de los conmutantes entre, por ejemplo, los sectores productivos, el tipo de cualificación, etc. Dado que el resultado está comprendido entre el valor 0 y el 100, puede también ser entendido e interpretado como un porcentaje. Por ejemplo, un índice de similitud temporal de 75 indicaría que dos matrices se parecen en 75\%; así, por ejemplo, esto significaría para este trabajo que la distribución de conmutantes laborales entre los dos años se parece en $75 \%$.

$$
\text { Coeficiente_de_especialización }=\frac{1}{2} \sum\left\{A B S\left[\frac{V_{i j}}{\sum j V_{i j}}-\frac{\sum i V_{i j}}{\sum i \sum j V_{i j}}\right]\right\}
$$

donde: $A B S$ indica valor absoluto; $V$ indica el valor de la variable; subíndice $i$ hace referencia al sector económico; subíndice $j$ hace referencia a un territorio.

$$
\text { Índice_de similitud_estructural }{ }^{a-b}=100\left[1-\frac{1}{2} \sum_{i=1}^{n+1} A B S\left[a_{i j}^{a}-a_{i j}^{b}\right]\right] p
$$

donde: $A B S$ indica valor absoluto; los súper índices $a y b$ hacen referencias a las economías, sectores o regiones; $n$ indica el número de sectores o regiones; $a_{i j}$ representa los coeficientes técnicos; $p$ representa el ponderador para corregir el tamaño de las economías.

$$
\text { Indice_de_similitud_temporal }^{(M 2-M 1)}=100\left[1-\frac{1}{2} \sum\left\{A B S\left[\frac{V_{i j}^{M 2}}{\sum_{i=1}^{n} \sum_{j=1}^{k} V_{i j}^{M 2}}-\frac{V_{i j}^{M 1}}{\sum_{i=1}^{n} \sum_{j=1}^{k} V_{i j}^{M 1}}\right]\right\}\right]
$$

donde: $A B S$, valor absoluto; $M 1$ hace referencia a la matriz de conmutantes en el periodo 1; $M 2$ hace referencia a la matriz de conmutantes en el periodo 2; Vij hace referencia al valor de la celda de la fila de la variable $i$ y la columna de la variable $j ; n$ hace referencia al número de filas; $k$ hace referencia al número de columnas. Para los efectos del presente trabajo, $M 1$ se refiere al año 2011; $M 2$ será el año 2016; los subíndices $i$ o $j$ pueden hacer referencia a las variables centro nodal principal, tipo de conmutación, sector productivo o tipo de formación. 


\section{Resultados}

En lo que sigue, en primer lugar, se proporcionan algunos antecedentes específicos para entender el fenómeno de la conmutación en el periodo de análisis, tal como los precios del transporte y la vivienda, además de las cifras de desocupación en el territorio de estudio. Posteriormente, se describen características de los tipos de conmutantes de acuerdo a los datos del año 2016 y se identifican diferencias estadísticas entre ellos. Finalmente, se caracteriza a la conmutación por medio de la metodología propuesta, esto es, haciendo uso del índice de similitud intertemporal, las tablas de contingencia y los gráficos circulares de Krzywinski et al. (2009).

En el Cuadro 1 se puede observar que el crecimiento de la conmutación laboral hacia los cinco centros nodales principales de la Patagonia chilena supera al crecimiento del total de ocupados en el periodo 2011-2016 (34.0\% versus $10.2 \%$ ). Distintos aspectos teóricos señalan que los costos de transporte podrían ayudar a explicar las decisiones de conmutación. Teniendo en cuenta este aspecto, desde datos del INE-Chile es posible calcular que el índice de precios al consumidor (IPC) tuvo una variación acumulada en el país de $19.1 \%$ entre diciembre de 2011 y diciembre de 2018 , mientras que los precios asociados al transporte aumentaron 9.2\%. Es decir, desde una perspectiva microeconómica, existió una disminución relativa de los precios del sector en relación a toda la canasta de bienes del índice de precios.

\section{Cuadro 1}

Variación de ocupados, conmutantes, índice de precios al consumidor (IPC). Años 2011-2016

\begin{tabular}{lccc}
\hline Descripción & 2011 & 2016 & $\begin{array}{c}\text { Variación } \\
\text { 2011-2016 }\end{array}$ \\
\hline Ocupados* & 346808 & 382290 & $10.2 \%$ \\
Conmutantes* & 18160 & 24342 & $34.0 \%$ \\
Variación, IPC** & & $19.1 \%$ \\
Variación precios en transporte** & & $11.1 \%$ \\
Variación precios arriendo viviendas** & & $28.4 \%$ \\
\hline
\end{tabular}

* Encuesta Suplementaria de Ingresos.

** Índice de precios al consumidor y sus divisiones de productos entre diciembre de 2011 y diciembre de 2016.

Fuente: Elaboración propia conforme a datos del INE-Chile, www.ine.cl 
En el Cuadro 1 también se puede observar que el precio del arriendo de viviendas (índice que pertenece a las subclases del IPC) para el mismo periodo creció $28.4 \%$ y representa tres veces el incremento del costo de transporte, hecho que también estaría reforzando el fenómeno de la conmutación. El precio del arriendo es un reflejo del precio de la tierra y se transfiere tanto a los precios de compra como al alquiler de propiedades, además de representar una proporción importante del presupuesto familiar (pago de hipoteca, alquiler o costo de oportunidad).

Es decir, existen dos elementos importantes que son planteados en la teoría y otros estudios empíricos que pueden estar asociados el fenómeno de la conmutación y que se han manifestado en el periodo de análisis de este trabajo: la efectiva disminución (relativa) de los costos de transporte y el efectivo incremento del valor de la tierra (incremento porcentual del valor del arriendo).

En el Cuadro 2 se pueden observar las tasas de desocupación proporcionadas por el INE-Chile para los años 2011 y 2016, tanto para el país como para las regiones político-administrativas que conforman la Patagonia. De lo anterior, se desprende con claridad que la desocupación anual en el territorio de estudio es más baja que en el resto del país y sólo existe la excepción en 2011, año en que la región de Los Ríos muestra un indicador más alto, destacando en el año 2016 cuando las regiones de Los Lagos y Aysén llegan a tener una desocupación que representa menos de la mitad de la tasa nacional.

\section{Cuadro 2}

Tasa de desocupación promedio anual.* Años 2011-2016

\begin{tabular}{llcc}
\hline Región & Centro nodal de análisis & $\mathbf{2 0 1 1}$ & $\mathbf{2 0 1 6}$ \\
\hline Los Ríos & Valdivia & 7.3 & 4.4 \\
Los Lagos & Osorno Puerto Montt & 4.5 & 2.7 \\
Aysén & Coyhaique & 4.1 & 2.9 \\
Magallanes y Antártica & Punta Arenas & 4.8 & 4.0 \\
Chilena & & 7.1 & 6.5 \\
País & & 7 & \\
\hline
\end{tabular}

* Corresponde al promedio de la tasa de desocupación de los trimestres móviles enero-marzo, abril-junio, julio-septiembre y octubre-diciembre de cada año.

Fuente: Elaboración propia conforme a datos del INE-Chile, www.ine.cl.

Este clásico indicador sintético del mercado del trabajo explicará lo que más adelante se expondrá: que gran parte de la conmutación proviene 
de la misma región político-administrativa, ya que los trabajadores no requerirían ir a otras regiones porque existen oportunidades de empleo en el territorio. Luego, seguirá en importancia la conmutación extrarregional, la que hemos definido como la que proviene desde fuera de la Patagonia, donde la desocupación es más alta y, por lo tanto, levemente alejada del pleno empleo.

Las características generales de los conmutantes se pueden encontrar en el Cuadro 3, en el que se detallan los tres tipos de conmutantes definidos: los intra, inter y extrarregionales, así como las características de los no conmutantes de los centros nodales. Desde el punto de vista de la edad promedio, aparentemente no existen diferencias entre los conmutantes y no conmutantes (columnas 4 y 5). Sin embargo, en un análisis comparado entre grupos, se puede identificar que los conmutantes intrarregionales, es decir aquellos que conmutan dentro de la misma región, son efectivamente más jóvenes que todos los demás grupos ( $p$-valor entre 0.06 y 0.002 ). Con los datos utilizados no es posible hacer una mayor inferencia de los datos, pero es plausible pensar que los individuos de menor edad promedio tienen menos experiencia laboral y generalmente están en una etapa de desarrollo individual donde el acceso a la vivienda es un anhelo personal y familiar, además de que, dados los antecedentes anteriores, ésta es más costosa en el periodo. Así, hipotéticamente, la conmutación vendría a solucionar la necesidad de trabajo y compatibilizar el acceso a la vivienda.

Desde el punto de vista del sexo de los ocupados, se observa con claridad que son proporcionalmente los hombres quienes conmutan en una mayor proporción que las mujeres y que, por tanto, es una variable relevante para entender la conmutación. Las diferencias son significativas al 1\%, tanto entre conmutantes como no conmutantes, en todas las comparaciones entre grupos. Al apreciar los resultados por tipo de conmutación, se desprende además que, a mayor distancia, mayor es la proporción de hombres, llegando incluso al $90.9 \%$ en los conmutantes extrarregionales. Este resultado es concordante con Roberts y Taylor (2016), donde se indica que los hombres están efectivamente más dispuestos a viajar distancias más largas, ya que se muestran más sensibles a las tasas de desempleo de su lugar de origen que las mujeres.

En relación con el salario promedio por hora, se descubren ciertos fenómenos. En primer lugar, existen diferencias significativas ( $\mathrm{p}$-valor $<0.01$ ) entre el salario de los conmutantes y los no conmutantes, siendo los primeros quienes ganan aproximadamente $30 \%$ más por hora (columnas 4 y 5). Sin embargo, tal diferencia estadística no existe entre los conmutantes intrarregionales y los no conmutantes, es decir los trabajadores de la mis- 
ma región, sean conmutantes o no, ganan el mismo salario por hora. Además, entre los conmutantes inter y extrarregionales, es decir, entre quienes se mueven en regiones de la Patagonia y quienes provienen desde fuera de ella, tampoco existen diferencias salariales por hora trabajada. Así, se desprende que son estos últimos los que efectivamente obtienen una mayor retribución salarial por hora, lo que, además, vendría a compensar los costos de la conmutación.

\section{Cuadro 3}

Características generales de los conmutantes y no conmutantes de los principales centros de la Patagonia. Año 2016

\begin{tabular}{lccccc}
\hline \multicolumn{5}{c}{ Conmutantes } \\
\hline & $\begin{array}{c}\text { Intra- } \\
\text { regionales } \\
\text { (1) }\end{array}$ & $\begin{array}{c}\text { Inter- } \\
\text { regionales } \\
\text { Características }\end{array}$ & $\begin{array}{c}\text { Extra- } \\
\text { regionales } \\
\text { (2) }\end{array}$ & $\begin{array}{c}\text { Total } \\
\text { conmutantes }\end{array}$ & $\begin{array}{c}\text { No } \\
\text { conmutantes } \\
\text { (5) }\end{array}$ \\
\hline $\begin{array}{l}\text { Edad promedio } \\
\begin{array}{l}\text { Porcentaje } \\
\text { hombres }\end{array}\end{array}$ & 39.3 & 45.7 & 41.7 & 40.6 & 43.7 \\
$\begin{array}{l}\text { Porcentaje } \\
\text { mujeres }\end{array}$ & $49.0 \%$ & $84.3 \%$ & $90.9 \%$ & $64.8 \%$ & $54.2 \%+++$ \\
$\begin{array}{l}\text { Salario } \\
\text { promedio hora } \\
\text { (dólares EU)* }\end{array}$ & $51.0 \%$ & $15.7 \%$ & $9.1 \%$ & $35.2 \%$ & $45.8 \%+++$ \\
\hline
\end{tabular}

+++ Indica que hay una diferencia significativa al $1 \%$ de la característica entre conmutantes y no conmutantes, utilizando la prueba de Kruskal-Wallis.

* Se calculó en primer lugar el salario en pesos chilenos, posteriormente se convirtió a dólares estadounidenses utilizando el valor del tipo de cambio observado promedio de 2016, publicado por el Banco Central de Chile, www.bcentral.cl

Fuente: Elaboración propia conforme a ESI del INE, www.ine.cl

El Cuadro 4 proporciona los resultados de la prueba Chi-2 aplicada a las tablas de contingencia (dimensiones) de interés que incluyen el CNP, el tipo de conmutación, los sectores productivos y el nivel de formación de los trabajadores conmutantes. En el mismo cuadro se especifica el anexo donde se encuentran los valores para realizar el cálculo del estadístico. Se observa que, para ambos años y para todas las dimensiones, el valor de Chi-2 es mayor que el valor de Chi-2 crítico; más específicamente, el p-valor en todos los casos es inferior al 1\%, lo que revela que existe una alta significancia estadística, confirmándose que no existe independencia entre las variables utilizadas para cada dimensión. Dados estos resultados, se podrá realizar un 


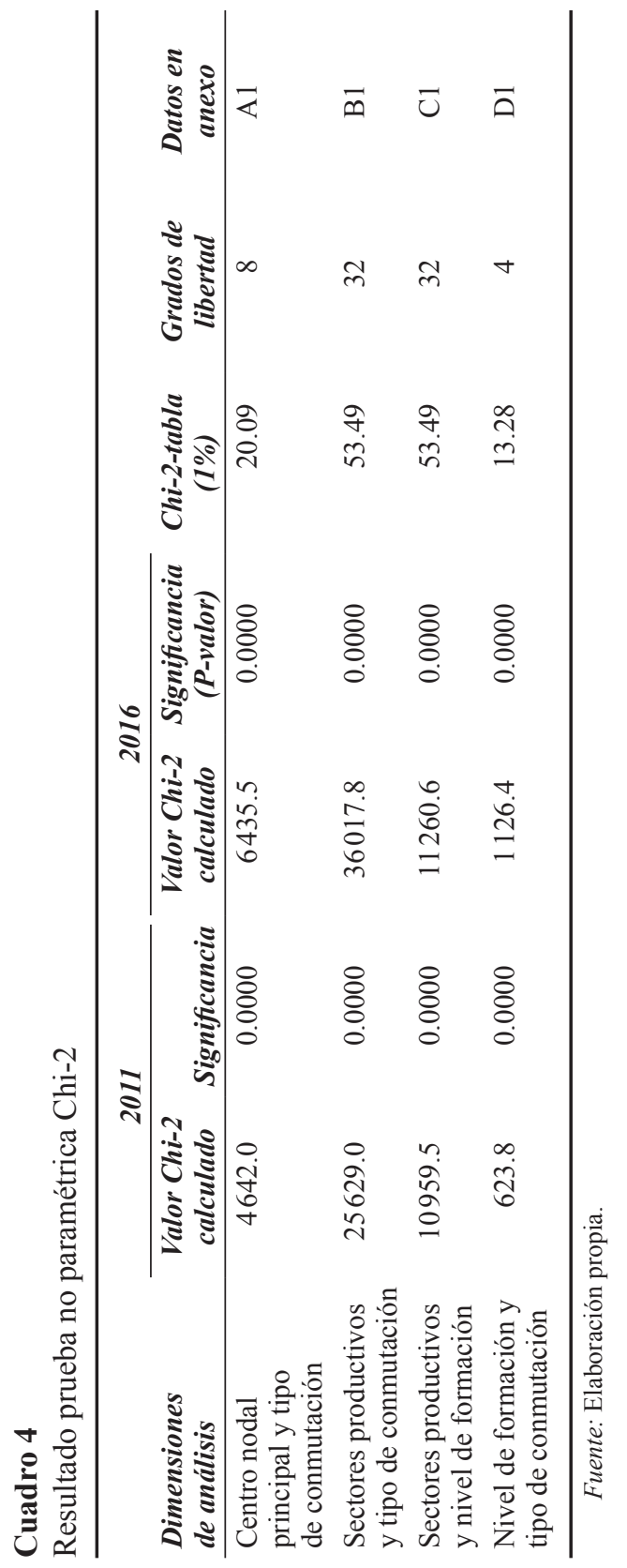


análisis respecto de los valores de la distribución de los conmutantes según las variables de análisis; es decir, se pueden analizar y describir los resultados que existen dentro de las tablas de contingencia.

El Cuadro 5 proporciona los resultados de los valores del índice de similitud para las cuatro dimensiones de análisis o cruces entre variables. Los valores para calcular el índice se encuentran en los anexos, y en el cuadro señalado se especifica la ubicación de ellos. Considérese el resultado del índice de similitud temporal para el CNP y el tipo de conmutación como ejemplo para comprender el análisis del índice. El resultado indica que entre los años 2011 y 2016 existió $66.27 \%$ de similitud entre la forma en cómo se distribuyen los conmutantes entre los centros nodales y el tipo de conmutación que realizan (sea ésta de tipo intrarregional, interregional o extrarregional). Desde el cuadro se observa que la dimensión que incluye el nivel de formación de los conmutantes (educación secundaria, técnica o profesional) y el sector productivo es la que más cambios experimentó, ya que su índice es de 52.01, correspondiendo a la distribución de los conmutantes entre variables que menos se parece entre los años. Del lado contrario, la distribución entre el tipo de conmutantes y el nivel de formación tiene el más alto parecido (índice de 79.72) entre los dos periodos. En lo que sigue de la presente sección de resultados, se proporcionan más detalles de las dimensiones aquí mencionadas.

\section{Cuadro 5}

Índice de similitud temporal de distribución de conmutantes por tipo de variables entre los años 2011 y 2016

\begin{tabular}{lcc}
\hline Dimensiones de análisis & $\begin{array}{c}\text { Índice } \\
\text { de similitud }\end{array}$ & $\begin{array}{c}\text { Datos } \\
\text { en anexo }\end{array}$ \\
\hline $\begin{array}{l}\text { Centro nodal principal (CNP) y tipo } \\
\text { de conmutación }\end{array}$ & 66.27 & $\mathrm{~A} 2$ \\
Tipo de conmutación y sectores productivos & 57.54 & $\mathrm{~B} 2$ \\
Sector productivo y nivel de formación & 52.01 & $\mathrm{C} 2$ \\
Nivel de formación y tipo de conmutación & 79.72 & $\mathrm{D} 2$ \\
\hline
\end{tabular}

Fuente: Elaboración propia.

En la Gráfica 1 es posible apreciar a los centros nodales principales y el origen de los conmutantes. Se observa que las cintas unen dos categorías o variables: los centros nodales principales de la Patagonia chilena y el origen de la conmutación. A su vez, el grosor de las cintas refleja la magnitud del flujo de conmutantes entre las categorías de las variables, por lo que los 
tamaños de cada una son comparables entre sí. De esta manera, en términos gráficos, una cinta más ancha representa un número de conmutantes más elevado en comparación con una más delgada.

\section{Gráfica 1}

Centros nodales principales y origen de la conmutación laboral

Año 2011

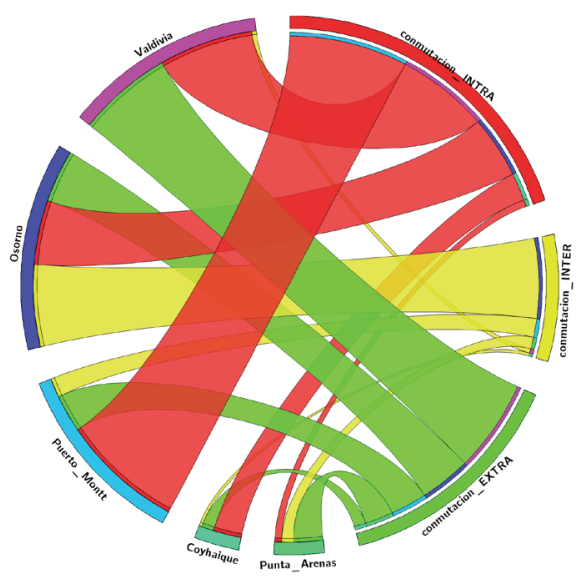

Año 2016

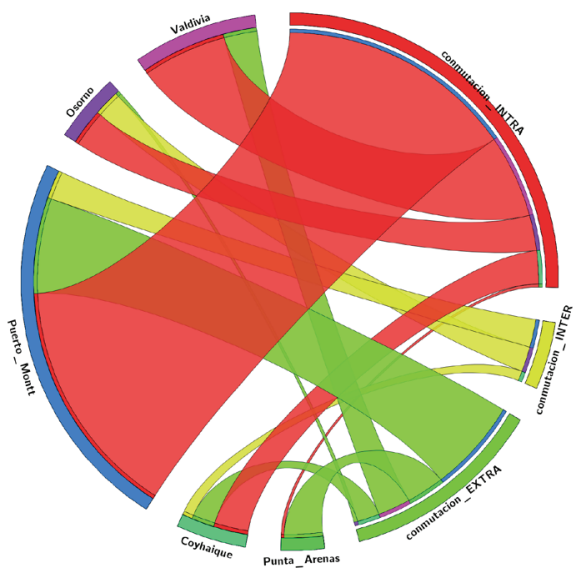

Fuente: Elaboración y análisis propio con datos de la Encuesta Suplementaria de Ingresos, años 2011 y 2016. 
Una forma de ver la gráfica y sus categorías es leerla en el sentido de las agujas del reloj. Por ejemplo, si se observa la conmutación intrarregional (para los años 2011 y 2016), la primera cinta de esta categoría se vincula con Puerto Montt, lo que quiere decir que el mayor flujo de conmutantes de este tipo va hacia ese centro nodal. Por otra parte, si se observa a Puerto Montt, coincide con que la primera cinta que llega hacia dicho centro nodal corresponde a los conmutantes del tipo intrarregionales. En cambio, para el caso de Osorno en el año 2011, el primer flujo de conmutantes correspondía a los de tipo interregional, es decir, los que provienen desde otras regiones de la misma Patagonia.

En la misma Gráfica 1 se aprecia que el principal tipo de conmutantes, dada la magnitud de la categoría, es el intrarregional, es decir los de corta distancia (provenientes del interior de las mismas regiones político-administrativas), lo que es respaldado con los resultados de la prueba Chi-2 (Cuadro 4), observándose una asociación entre las dos variables. No obstante, y contrario a lo esperado, el segundo flujo en importancia para ambos años corresponde al que proviene desde fuera de la Patagonia. Este resultado determina que la primera de las hipótesis, la que indicaba que existiría una relación inversa entre la distancia y el flujo de conmutantes hacia los centros nodales principales de la Patagonia chilena, sea refutada.

Para la dimensión del análisis anterior, el índice de similitud temporal propuesto en este trabajo arroja como resultado un valor de 66.27, es decir, existiría algún grado de similitud en la distribución del tipo de conmutantes de los CNP entre los años 2011 y 2016, así como diferencias destacables. En primer lugar, en la misma Gráfica 1 se observa que la conmutación entre las regiones de la Patagonia (la interregional) pierde peso relativo, mientras que la que incrementa su importancia es la de corta distancia (intrarregional). Un segundo aspecto relevante es lo que sucede con los centros Puerto Montt y Punta Arenas. Para el primero, se observó un incremento en términos de recepción de conmutantes; el caso de Punta Arenas destaca por el incremento de la conmutación del tipo extrarregional, a tal punto que su vínculo laboral (desde el punto de vista de un centro receptor) es casi nulo con las otras regiones de la Patagonia para el año 2016.

Otro aspecto que destaca es el hecho de que Osorno sea para el año 2011 el CNP más conectado con el resto de las regiones de la Patagonia, es decir, el que recibe, proporcionalmente, más conmutantes interregionales; sin embargo, el 2016 pierde parte de estos conmutantes, además de importancia relativa.

En resumen, se observa que existe una alta participación territorial de conmutantes que viven cerca de los CNP, pero aislados laboralmente con 
respecto al resto de la Patagonia. Recuérdese, además, que al inicio de esta sección se señaló que las bajas tasas de desocupación en la Patagonia podrían explicar en parte que la conmutación proveniente desde la misma región político-administrativa sea la más importante, ya que, aparentemente, no sería necesario que los trabajadores vayan hacia otras regiones en busca de empleo.

La segunda dimensión de análisis es la que se presenta en la Gráfica 2, donde el índice de similitud temporal es de 57.74. Este resultado indica el segundo menor cambio de distribución de los conmutantes entre los años de análisis, al tiempo que muestra diferencias a considerar. Para un mejor análisis, y dada la naturaleza de la gráfica y los nombres de las variables, ha sido necesario construir y proporcionar el Cuadro 6, que indica el significado de la simbología asociada a los sectores productivos de las figuras.

Los resultados de la prueba Chi-2 también permiten indicar que existe una asociación entre las variables, es decir, algún tipo de conmutación está más asociada a un tipo de sector económico. Se aprecia, para el año 2011, que los principales sectores productivos que más conmutantes reciben son construcción (19.1\%) y comercio (17.6\%), cuyos principales flujos de conmutantes correspondían a intrarregionales (47.6 y $43.1 \%$ respectivamente); sin embargo, en el año 2016 el principal flujo de conmutantes en el sector construcción provenía desde fuera de la Patagonia (62.6\%), y el sector comercio aumentó considerablemente la proporción de conmutantes intrarregionales a $84.2 \%$.

En cambio, en el año 2016 los sectores más importantes desde el punto de vista de la conmutación son: transporte (16.1\%), pesca (12.7\%) e industria manufacturera (12.6\%). En este último, el principal flujo de conmutantes es del tipo intrarregional; para los otros dos, extrarregional.

$\mathrm{Al}$ agrupar los sectores en primarios, secundarios y terciarios entre los años 2011 y 2016, los conmutantes en las actividades terciarias son los que tienen el mayor incremento porcentual, pasando de 50.4 a $61.8 \%$. Los resultados indican que las actividades que son atractivas para los trabajadores conmutantes en la Patagonia se desarrollan principalmente en los sectores terciario y secundario ( $80.7 \%$ en 2011 y $82.4 \%$ en 2016), siendo las menos atractivas aquellas vinculadas a los sectores primarios. Así, la conmutación laboral en la Patagonia cumple el patrón de búsqueda en sectores que, potencialmente, pagarían mayores ingresos. Este resultado permite confirmar la tercera hipótesis, que indicaba que la conmutación laboral en la Patagonia chilena estaría asociada principalmente a los sectores productivos terciario y secundario. 


\section{Gráfica 2}

Sectores productivos y origen de la conmutación

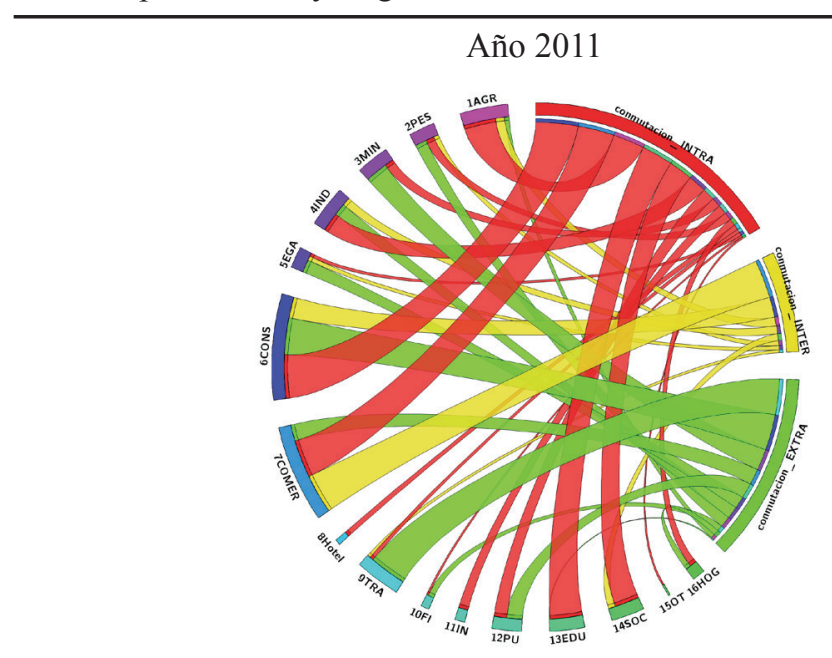

Año 2016

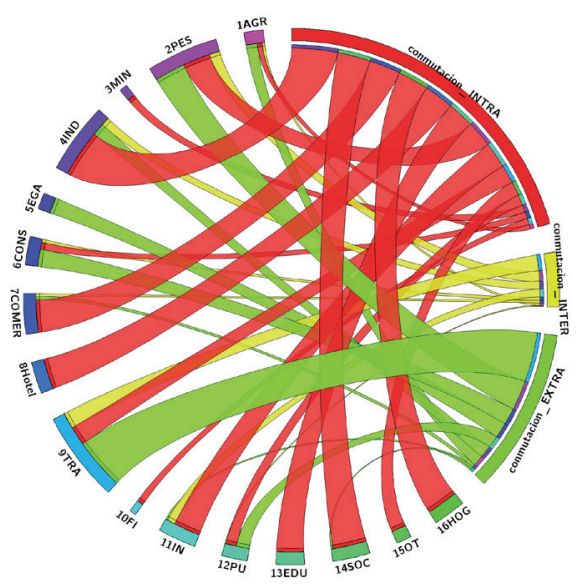

Fuente: Elaboración y análisis propio con datos de la Encuesta Suplementaria de Ingresos, años 2011 y 2016.

Como una forma de complementar antecedentes de los sectores productivos, en la Gráfica 3 se puede observar el tipo de formación de los conmutantes y hacia qué sectores productivos se trasladan. Dado los resultados 
de la prueba Chi-2, nuevamente se puede concluir que existe una asociación entre las variables para ambos años. A su vez, de acuerdo al índice de similitud temporal 52.01, se observa que corresponde a la dimensión de análisis que más variación tuvo entre los dos años.

\section{Cuadro 6}

Código de los sectores productivos en las gráficas

\begin{tabular}{ll}
\hline Código & \multicolumn{1}{c}{ Sector productivo } \\
\hline 1AGR & Agricultura, ganadería, caza y silvicultura \\
2PES & Pesca \\
3MIN & Explotación de minas y canteras \\
4IND & Industrias manufactureras \\
5EGA & Suministro de electricidad, gas y agua \\
6CONS & Construcción \\
7COMER & Comercio al por mayor y por menor; reparación vehículos \\
& automotores, motocicletas, efectos personales y enseres domésticos \\
8Hotel & Hoteles y restaurantes \\
9TRA & Transporte, almacenamiento y comunicaciones \\
10FI & Intermediación financiera \\
11IN & Actividades inmobiliarias, empresariales y de alquiler \\
12PU & Administración pública y defensa; planes de seguridad \\
& social de afiliación obligatoria \\
13EDU & Enseñanza \\
14SOC & Servicios sociales y de salud \\
15OT & Otras actividades de servicios comunitarios, sociales y personales \\
16HOG & Hogares privados con servicio doméstico \\
\hline
\end{tabular}

Fuente: Elaboración propia.

Concretamente, para 2011 se observa que el flujo de conmutantes con estudios técnicos y superiores se orienta a actividades productivas del tipo terciario ( 65.8 y $81.8 \%$ respectivamente), mientras que en el año 2016 destaca que, de aquellos que poseen estudios superiores, el $90 \%$ se orienta hacia sectores terciarios. Para el caso de los conmutantes ocupados con estudios técnicos, se observa un importante incremento porcentual de trabajadores que van hacia sectores primarios (pasan de 13.5 a $32.5 \%$ ). Desde la perspectiva del tipo de la cualificación, se puede mencionar al sector de intermediación financiera (11IN en la gráfica), ya que el 100\% de los conmutantes poseen estudios superiores en ambos periodos. 


\section{Gráfica 3}

Sectores productivos y nivel de formación
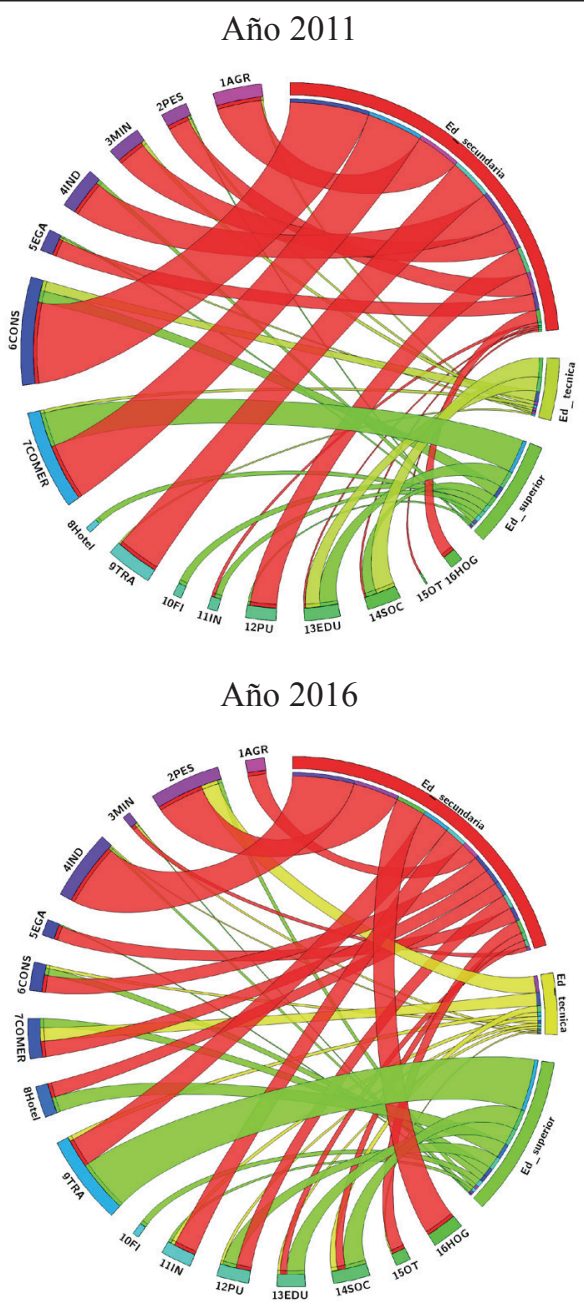

Fuente: Elaboración y análisis propio con datos de la Encuesta Suplementaria de Ingresos, años 2011 y 2016.

La última dimensión de análisis relaciona a las variables nivel de formación y tipo de conmutación. Al igual que en los casos anteriores, los resultados de la prueba Chi-2 permiten afirmar que existe una asociación de las 
variables. Por otra parte, por medio del índice de similitud temporal, es posible señalar que ésta fue la dimensión que menos cambió entre los dos años. Específicamente, la similitud entre las dos matrices fue de 79.72 por ciento.

Finalmente, luego de revisar la Gráfica 4, se puede observar que, para ambos años, el principal flujo de conmutantes sólo posee educación secundaria o menos, independientemente de la distancia y origen. En este sentido, el 79.4\% de los conmutantes extrarregionales en 2011 tenía estudios secundarios o un menor nivel, y aquellos que tenían estudios superiores representaban el 12.5\%; en cambio, para el año 2016 estos últimos ahora representaban el $38.9 \%$. A pesar del incremento anterior, los resultados no permiten confirman la segunda hipótesis de trabajo, que indicaba que la conmutación de más larga distancia estaría asociada a niveles más altos de formación, ya que, incluso incorporando a los trabajadores con estudios técnicos, sólo alcanzan conjuntamente al $44.8 \%$. Sin embargo, el incremento de conmutantes extrarregionales con mayor cualificación también podría estar vinculado con el mejor desempeño del mercado laboral en la Patagonia, por lo que trabajadores desde fuera de esta región podrían estar buscando alternativas laborales que no encuentran en sus regiones de origen.

\section{Conclusiones}

Incorporando una perspectiva intermedia en la conmutación laboral, y con una baja representatividad de los datos existentes para analizarla, este trabajo contribuye a proponer un diseño metodológico, con datos agrupados, formulando un índice de similitud temporal con aplicación de una prueba no paramétrica que puede ser aplicada a otros territorios, en especial cuando se dan condiciones como falta de información o de representación estadística.

Concretamente, el índice de similitud temporal ayuda de forma sintética a leer los cambios en los patrones de distribución de los conmutantes entre distintas dimensiones. Específicamente, se aplicó el procedimiento para analizar la conmutación laboral que ha existido en los cinco centros nodales principales de la Patagonia chilena entre los años 2011 y 2016. Los resultados permiten concluir que existen diferencias en el periodo, pero también se hace necesario realizar análisis con un periodo mayor de tiempo.

Para el territorio específico, y con el procedimiento adoptado, los hallazgos indican que la conmutación laboral tiene distintos patrones entre los CNP desde el punto de vista de su origen en los diferentes años. Así, en la Patagonia chilena predomina la del tipo intrarregional, de corta distancia, seguida por la del tipo extrarregional, de más larga distancia. Se concluye 
que los CNP están poco conectados, desde el punto de vista de la conmutación laboral, con el resto de las regiones de la Patagonia.

\section{Gráfica 4}

Niveles de formación y origen de la conmutación

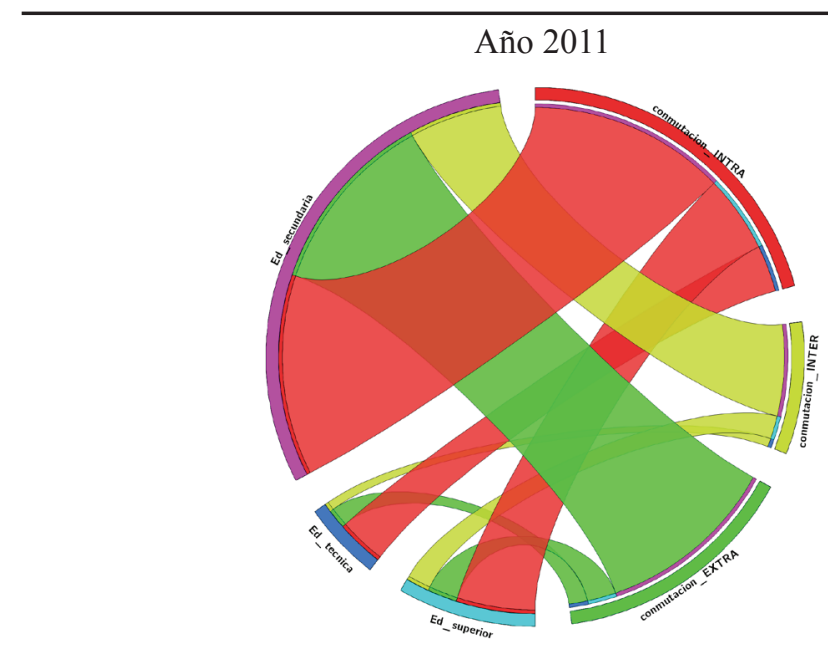

Año 2016

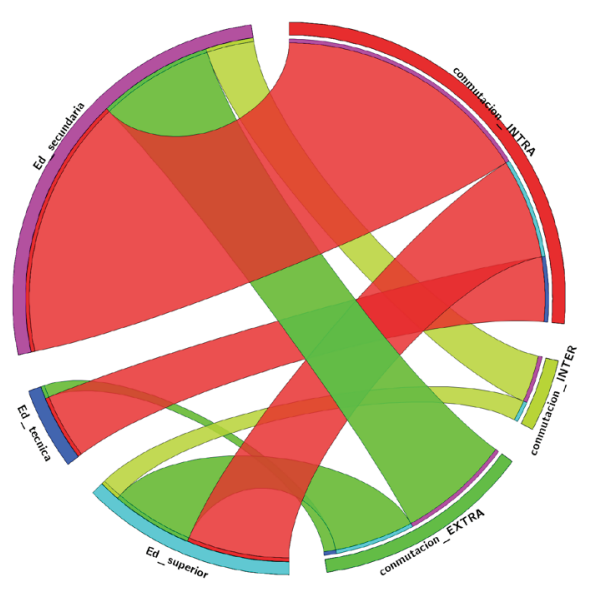

Fuente: Elaboración y análisis propio con datos de la Encuesta Suplementaria de Ingresos, años 2011 y 2016. 
Dado que el territorio analizado posee vínculos inesperados desde una perspectiva laboral, es posible concebir funcionalmente, y dada la conmutación existente, que los CNP son parte de dos regiones funcionales: el territorio más cercano al mismo centro nodal y una "región" que está fuera de la Patagonia. A partir de los hallazgos, es posible concebir a la Patagonia chilena como un territorio policéntrico, en sintonía con lo indicado por Yinger (1992), ya que existen varios centros que atraen conmutantes desde territorios cercanos (conmutación de corta distancia).

Los costos de transporte, que en términos relativos han disminuido en Chile, han contribuido a facilitar el crecimiento del fenómeno. Sin embargo, se observa que han existido importantes cambios temporales de comportamiento y características de los conmutantes, como su nivel de formación y los sectores productivos hacia los que conmutan. Así, se observó un aumento de la conmutación hacia sectores económicos secundarios y terciarios por sus mayores salarios en la Patagonia, en conjunto con los menores costos de transporte.

Los hallazgos también indican que las políticas públicas para territorios como la Patagonia chilena, que tengan como foco la mejora de la productividad de los sectores económicos, deben tener en consideración el tipo de cualificación de los conmutantes, ya que, de acuerdo a los resultados, se observa una alta conmutación de baja cualificación, a pesar de que en el año 2016 había existido un incremento de conmutantes con nivel técnico y superior. Así, las políticas públicas pueden contribuir a aumentar el capital humano por la vía de la formación y la capacitación, lo cual tiene efectos en el mediano o largo plazos, pero también deben considerar como una vía alternativa o complementaria la atracción de recursos humanos cualificados por medio de la conmutación para fortalecer los sectores productivos. 


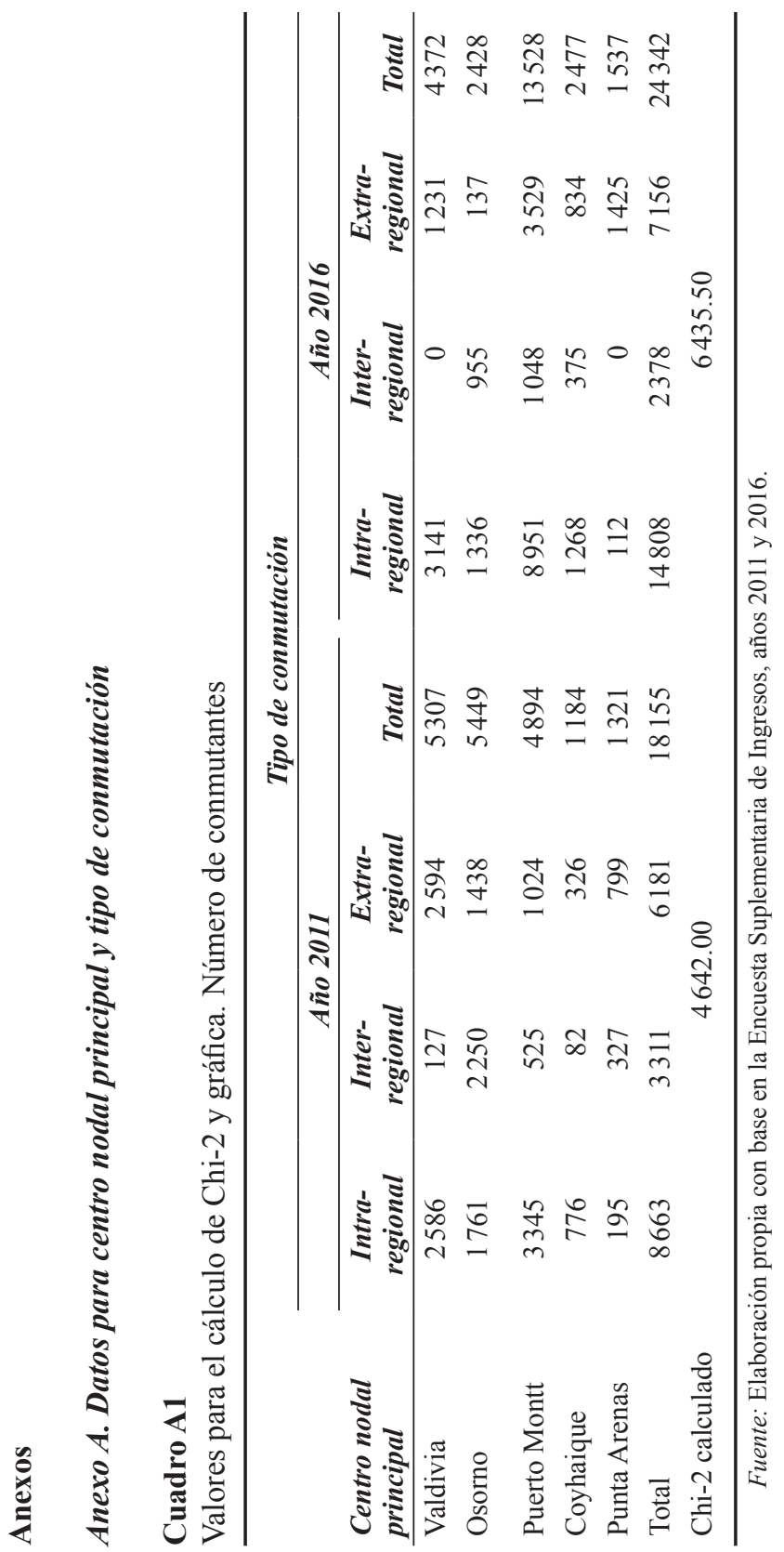




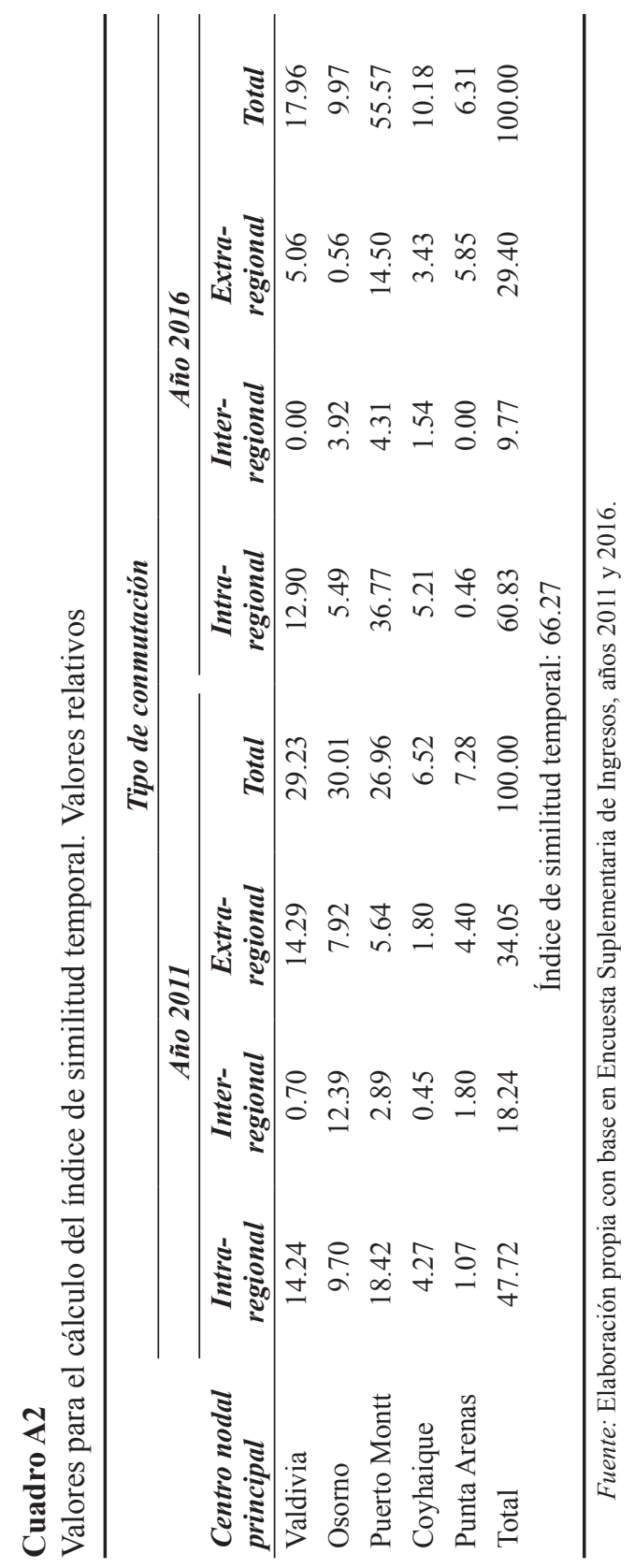




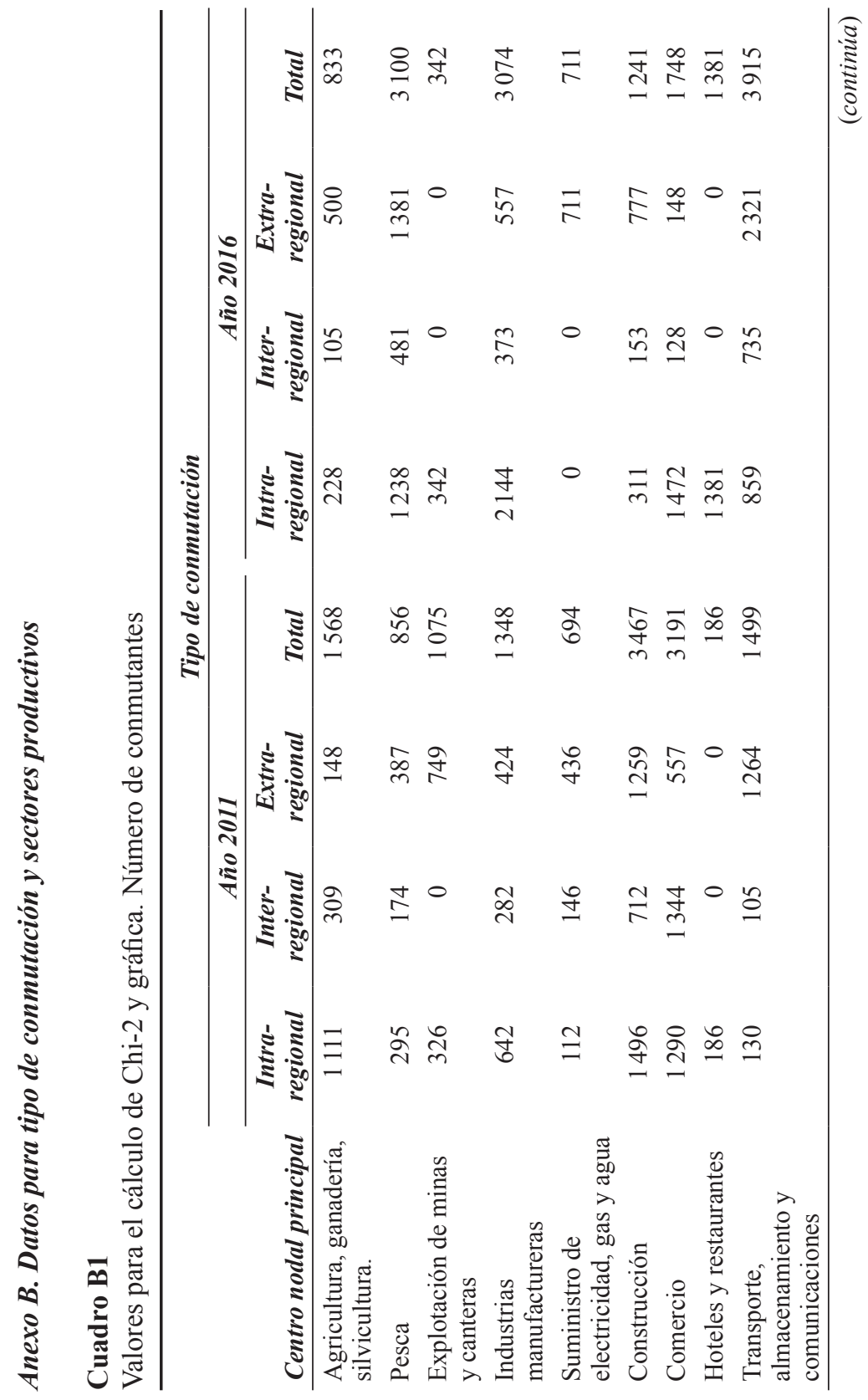




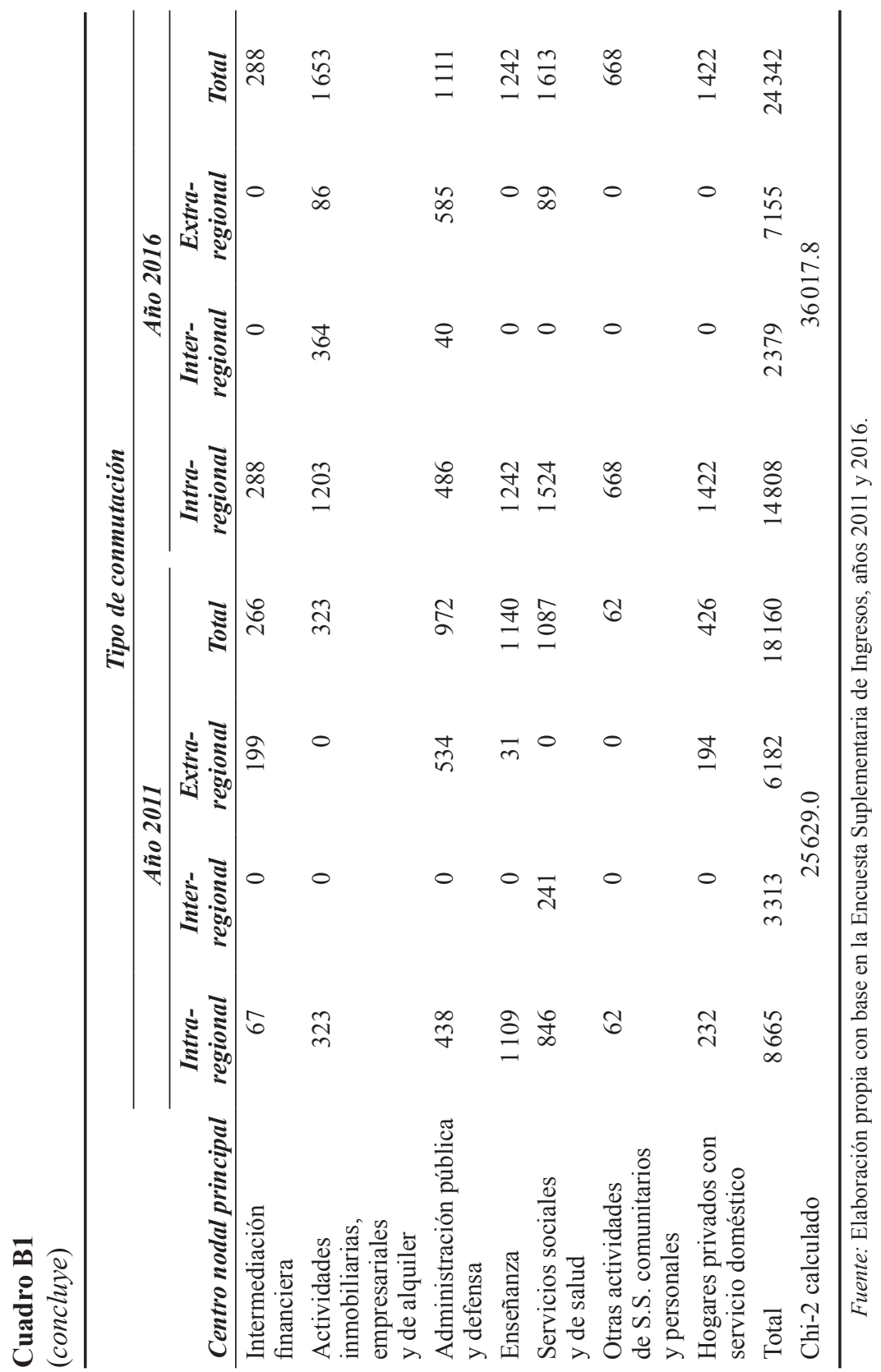




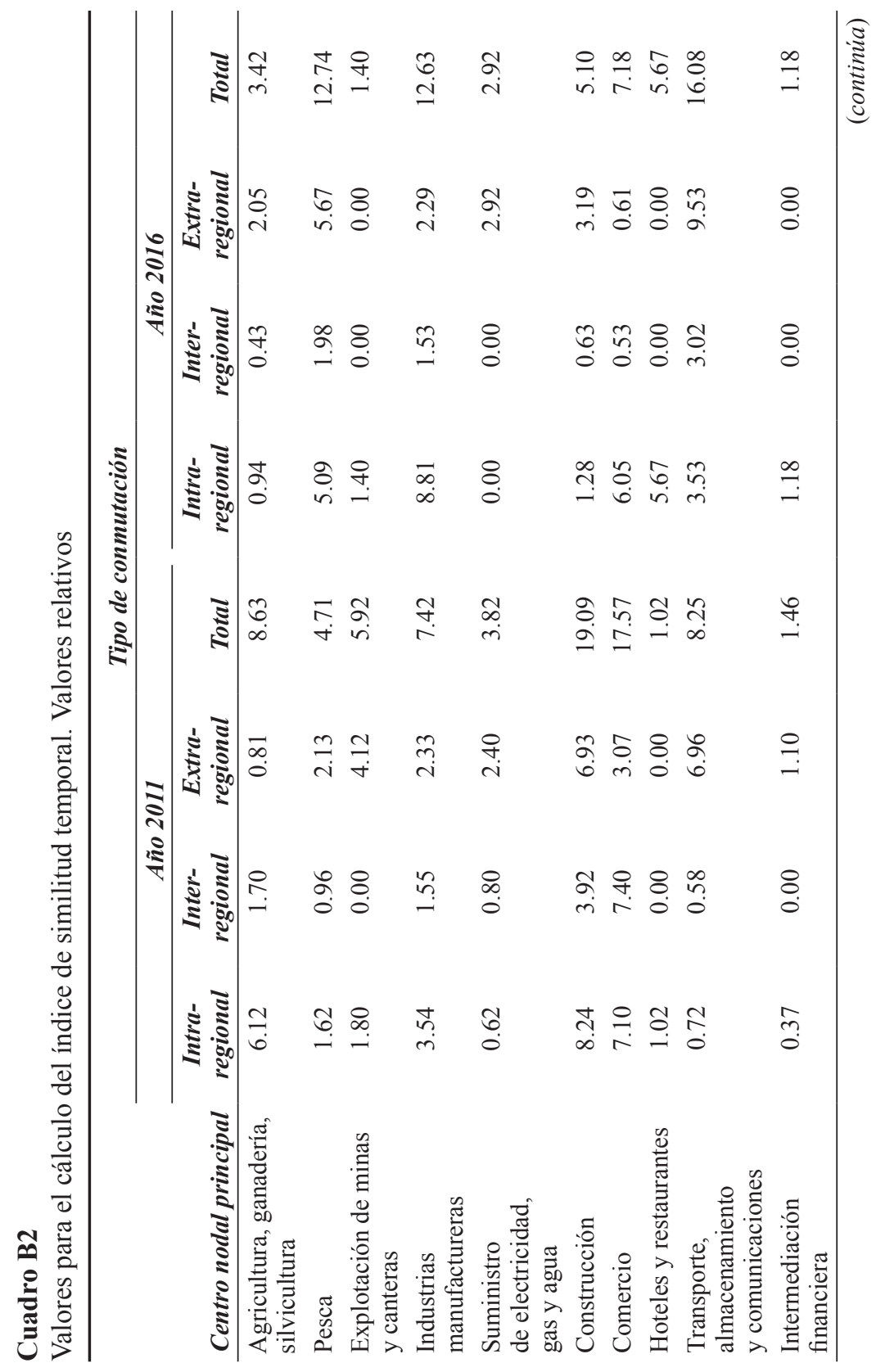




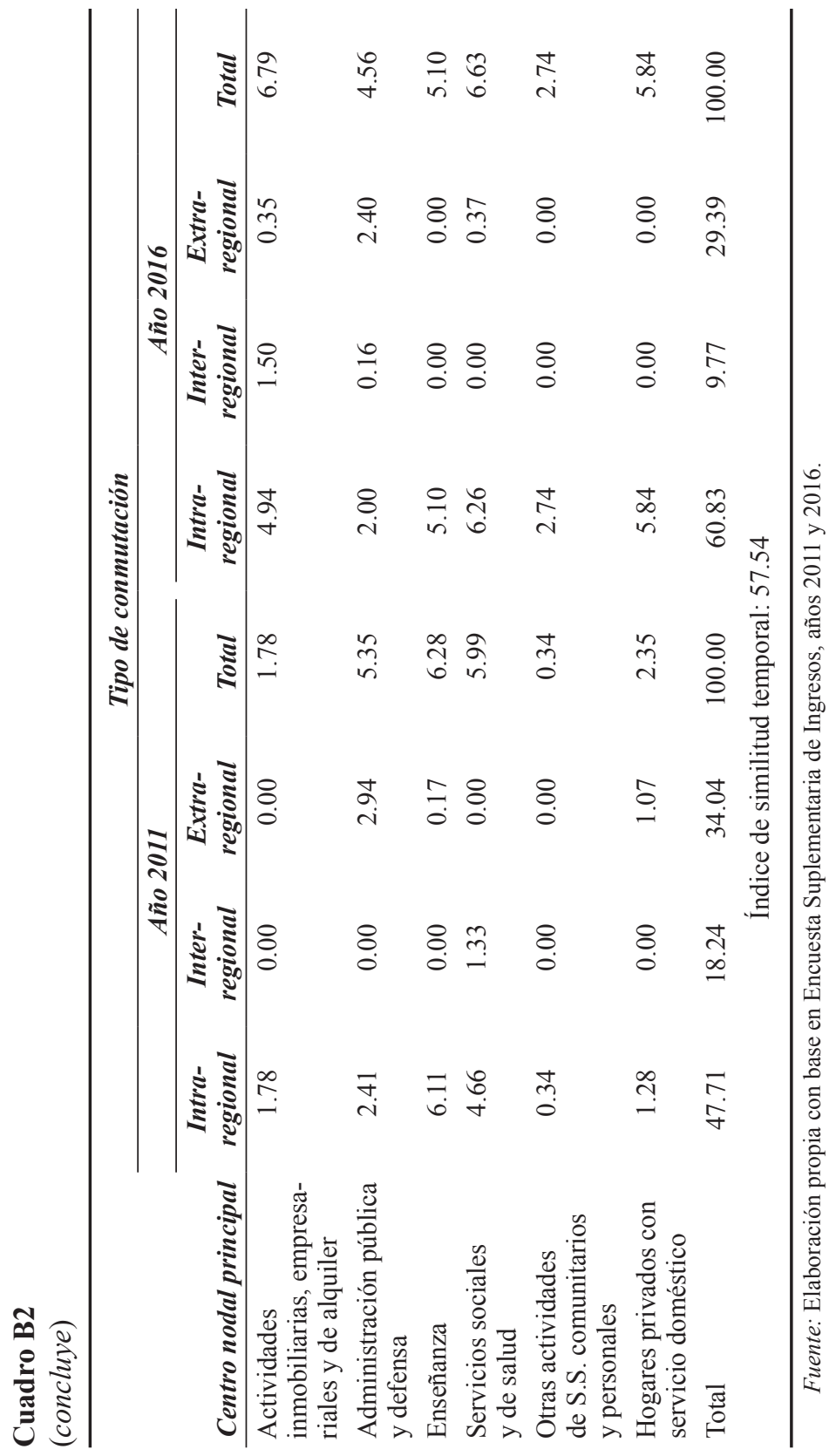




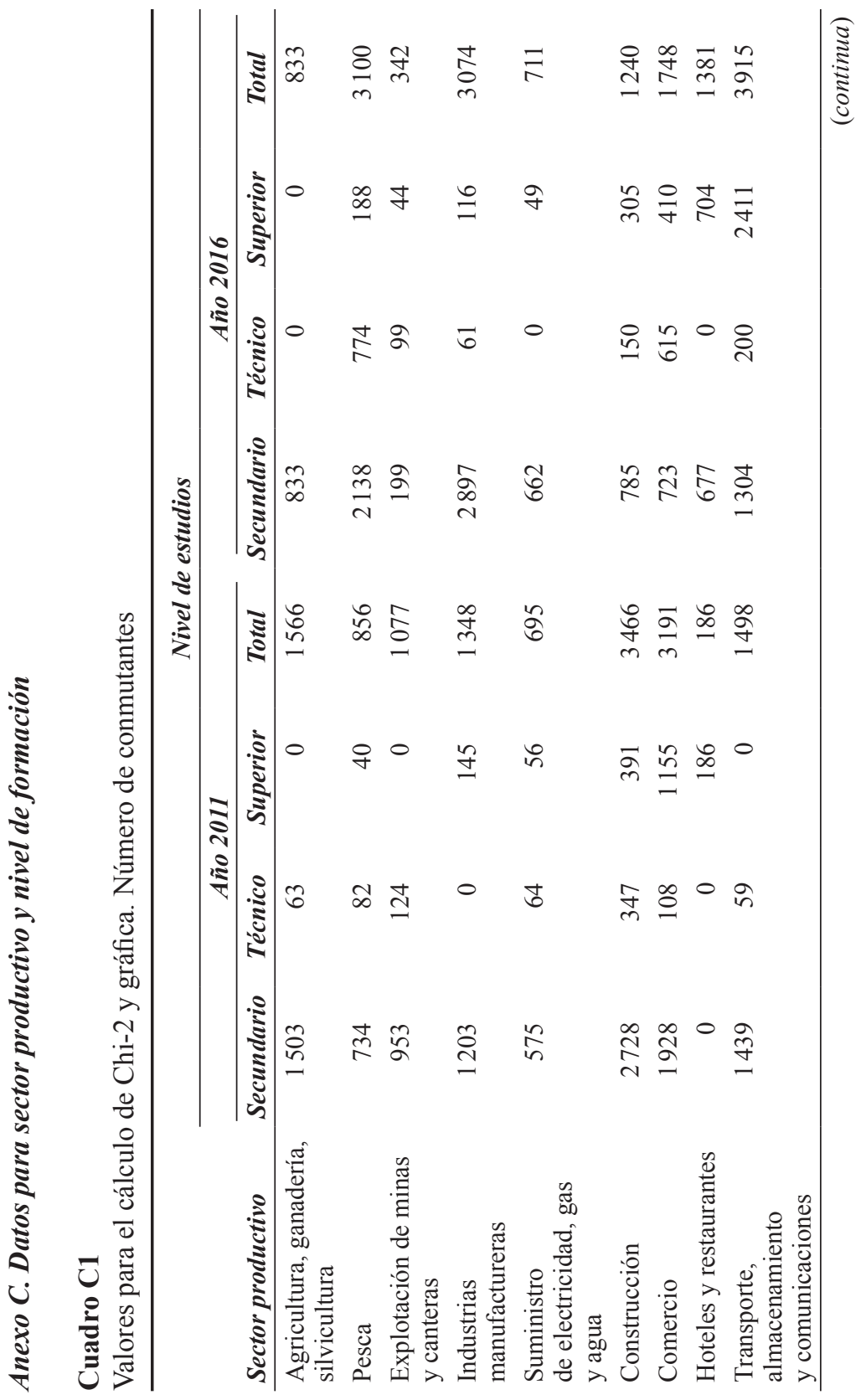




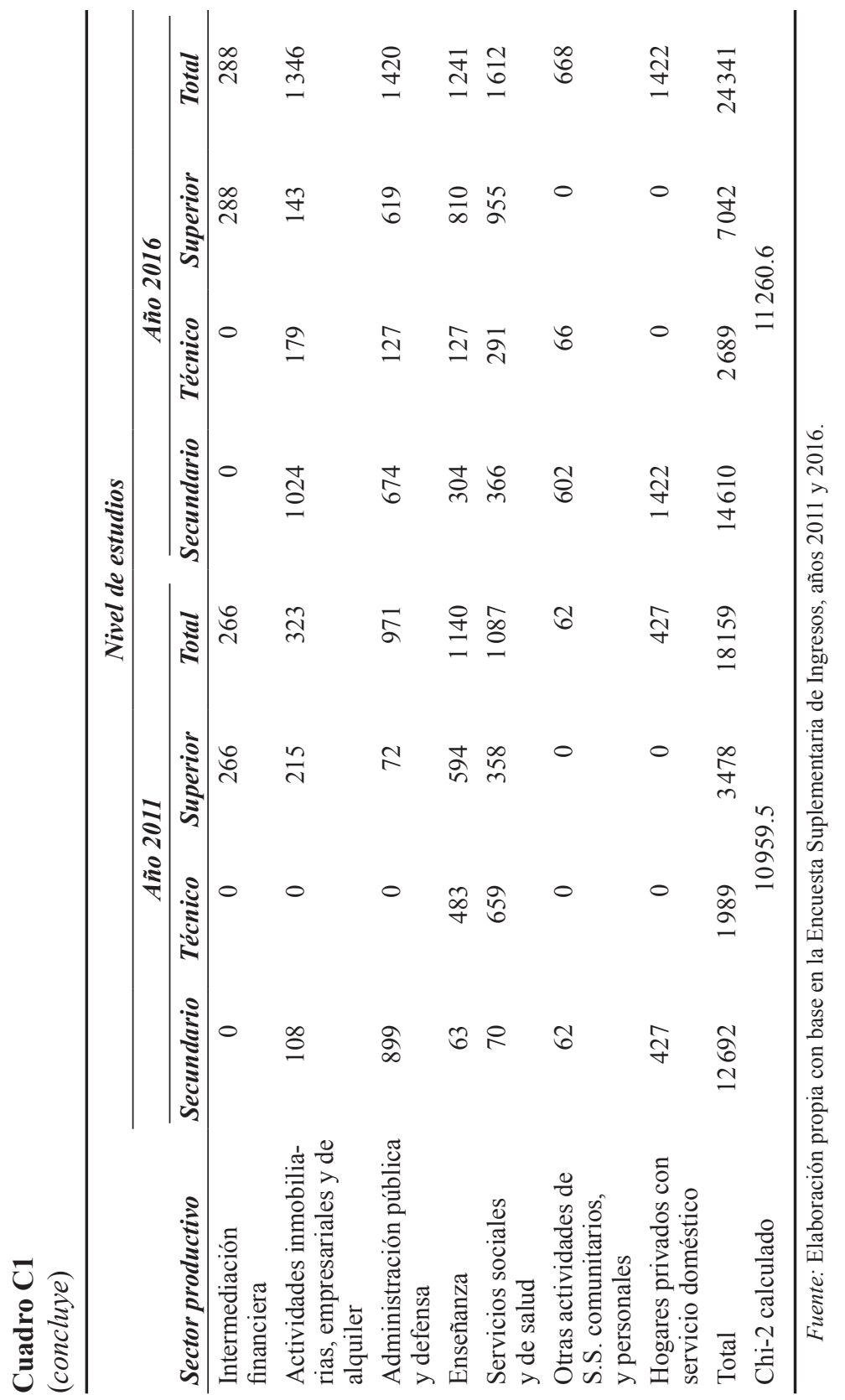




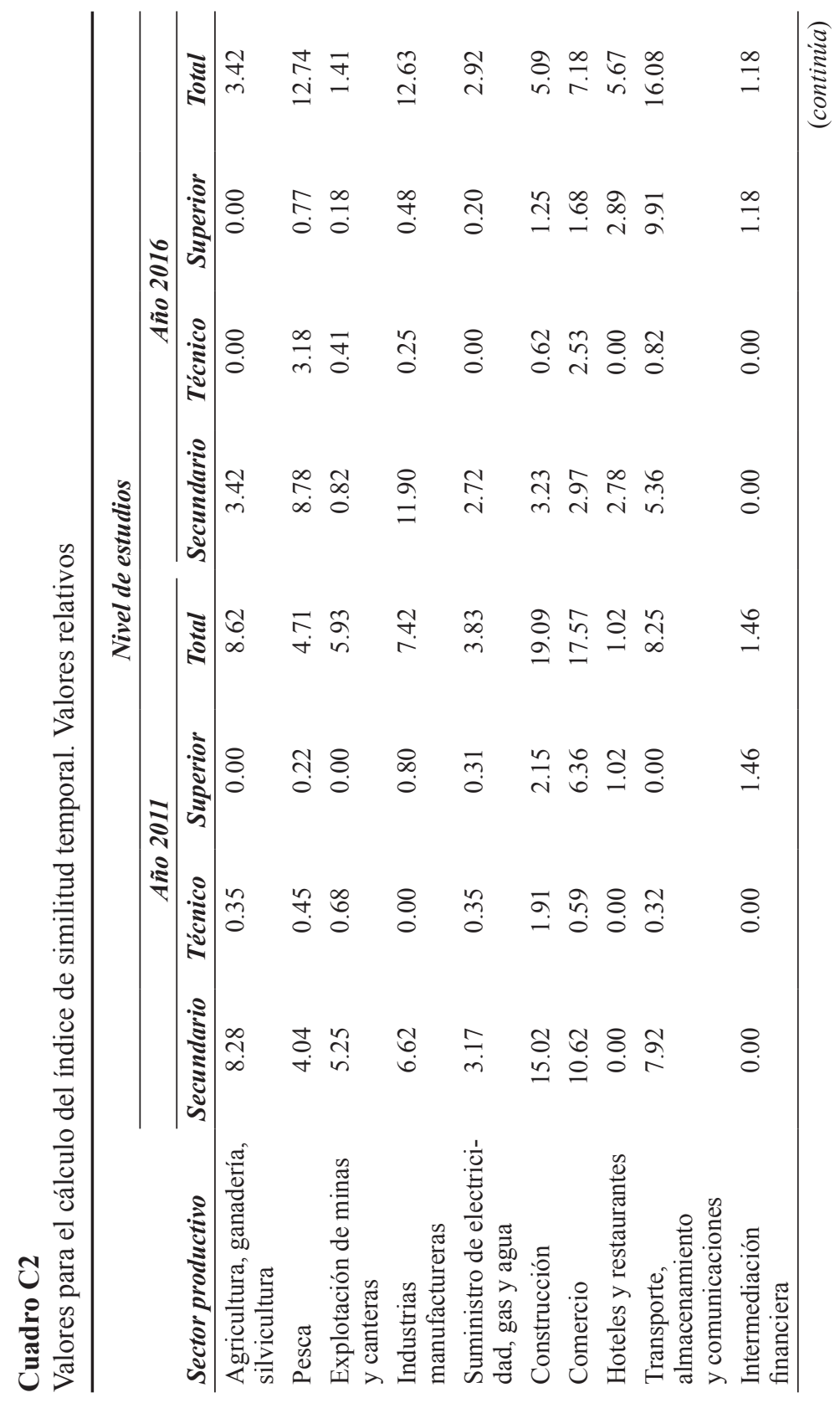




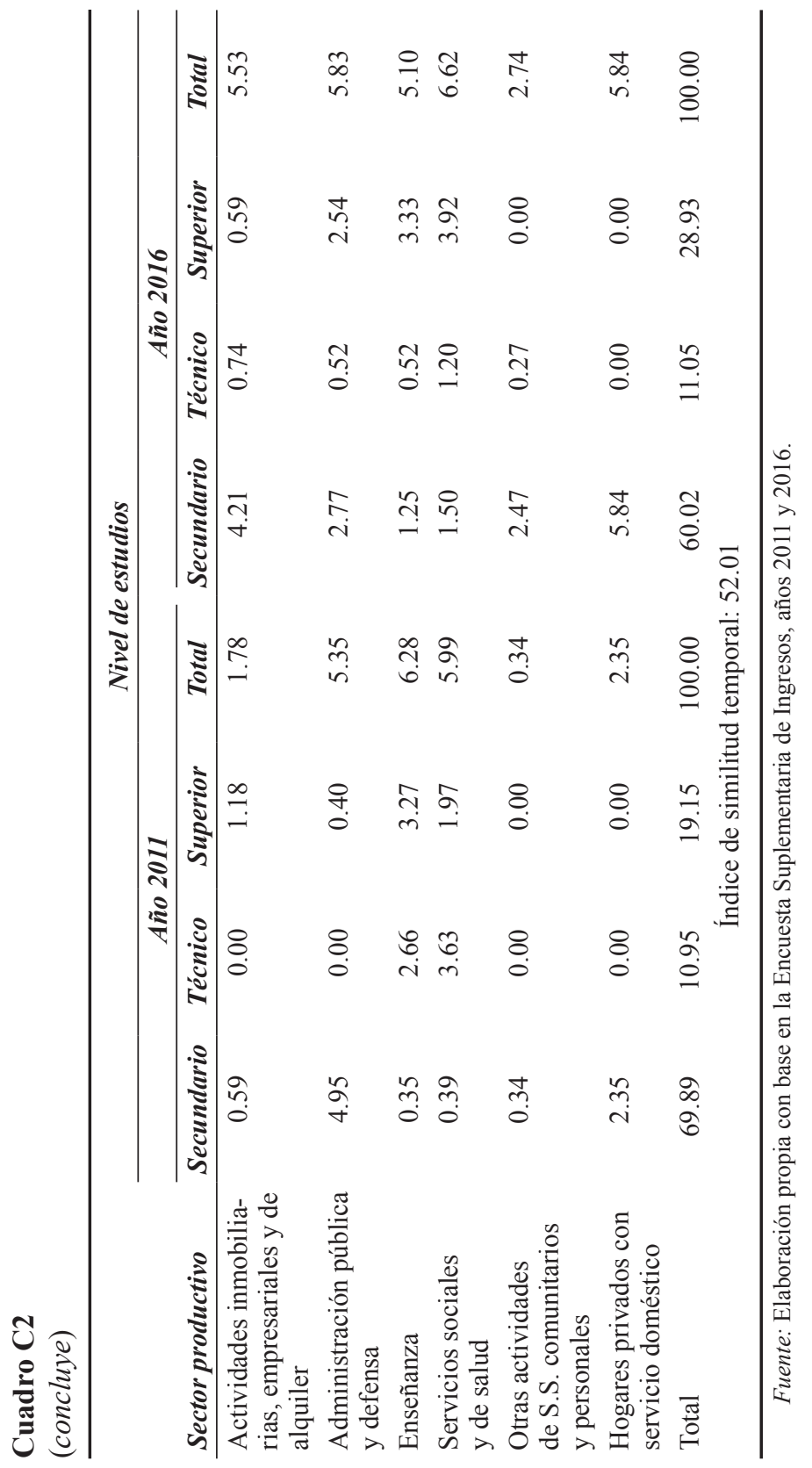




\section{Anexo D. Datos para nivel de formación y tipo de conmutación}

\section{Cuadro D1}

Valores para el cálculo de Chi-2 y gráfica. Número de conmutantes

\begin{tabular}{|c|c|c|c|c|c|c|c|c|}
\hline \multirow[b]{3}{*}{$\begin{array}{l}\text { Nivel de } \\
\text { estudios }\end{array}$} & \multicolumn{8}{|c|}{ Tipo de conmutación } \\
\hline & \multicolumn{4}{|c|}{ Año 2011} & \multicolumn{4}{|c|}{ Año 2016} \\
\hline & $\begin{array}{c}\text { Intra- } \\
\text { regional }\end{array}$ & $\begin{array}{c}\text { Inter- } \\
\text { regional }\end{array}$ & $\begin{array}{c}\text { Extra- } \\
\text { regional }\end{array}$ & Total & $\begin{array}{c}\text { Intra- } \\
\text { regional }\end{array}$ & $\begin{array}{c}\text { Inter- } \\
\text { regional }\end{array}$ & $\begin{array}{c}\text { Extra- } \\
\text { regional }\end{array}$ & Total \\
\hline Secundario & 5322 & 2462 & 4905 & 12689 & 9009 & 1650 & 3952 & 14611 \\
\hline Técnico & 1260 & 228 & 501 & 1989 & 2269 & 0 & 420 & 2689 \\
\hline Superior & 2081 & 621 & 775 & 3477 & 3530 & 728 & 2784 & 7042 \\
\hline Total & 8663 & 3311 & 6181 & 18155 & 14808 & 2378 & 7156 & 24342 \\
\hline $\begin{array}{l}\text { Chi-2 } \\
\text { calculado }\end{array}$ & \multicolumn{4}{|c|}{623.8} & \multicolumn{4}{|c|}{1126.4} \\
\hline
\end{tabular}

Fuente: Elaboración propia con base en la Encuesta Suplementaria de Ingresos, años 2011 y 2016.

\section{Cuadro D2}

Valores para el cálculo del índice de similitud temporal. Valores relativos

\begin{tabular}{|c|c|c|c|c|c|c|c|c|}
\hline \multirow[b]{3}{*}{$\begin{array}{l}\text { Nivel de } \\
\text { estudios }\end{array}$} & \multicolumn{8}{|c|}{ Tipo de conmutación } \\
\hline & \multicolumn{4}{|c|}{ Año 2011} & \multicolumn{4}{|c|}{ Año 2016} \\
\hline & $\begin{array}{c}\text { Intra- } \\
\text { regional }\end{array}$ & $\begin{array}{l}\text { Inter- } \\
\text { regional }\end{array}$ & $\begin{array}{c}\text { Extra- } \\
\text { regional }\end{array}$ & Total & $\begin{array}{l}\text { Intra- } \\
\text { regional }\end{array}$ & $\begin{array}{c}\text { Inter- } \\
\text { regional }\end{array}$ & $\begin{array}{c}\text { Extra- } \\
\text { regional }\end{array}$ & Total \\
\hline Secundario & 29.31 & 13.56 & 27.02 & 69.89 & 37.01 & 6.78 & 16.24 & 60.02 \\
\hline Técnico & 6.94 & 1.26 & 2.76 & 10.96 & 9.32 & 0.00 & 1.73 & 11.05 \\
\hline Superior & 11.46 & 3.42 & 4.27 & 19.15 & 14.50 & 2.99 & 11.44 & 28.93 \\
\hline Total & 47.72 & 18.24 & 34.05 & 100.00 & 60.83 & 9.77 & 29.40 & 100.00 \\
\hline \multicolumn{9}{|c|}{ Índice de similitud temporal: 79.72} \\
\hline
\end{tabular}

Fuente: Elaboración propia con base en la Encuesta Suplementaria de Ingresos, años 2011 y 2016. 


\section{Bibliografía}

Ábalos, M. y Paredes, D. (2014). Una modificación al método varimax para delimitar regiones urbanas funcionales usando la vecindad espacial. Investigaciones Regionales, 30, 103-126. Recuperado de https://dialnet. unirioja.es/servlet/articulo?codigo $=4965059$

Abel, G. y Sander, N. (2014). Quantifying global international migration flows. Science, 343(6178), 1520-1522. Recuperado de https://science. sciencemag.org/content/343/6178/1520.full

Alonso, W. (1960). A theory of the urban land market. Papers and Proceedings of the Regional Science Association, 6, 149-157. Recuperado de https:// rsaiconnect.onlinelibrary.wiley.com/doi/abs/10.1111/j.1435-5597.1960. tb01710.x

Aroca, P. y Atienza, M. (2007). Impacto sobre el crecimiento regional de la migración y conmutación interregional en Chile. Taller Nacional sobre Migración interna y desarrollo en Chile: diagnóstico, perspectivas y políticas, Comisión Económica para América Latina y el Caribe, División de Población. Recuperado de https://www.cepal.org/sites/default/ files/courses/files/paroca.pdf

Aroca, P. y Atienza, M. (2008). La conmutación regional en Chile y su impacto en la región de Antofagasta. Eure, Revista Latinoamericana de Estudios Urbano Regionales, 34(102), 97-120. Recuperado de https:// scielo.conicyt.cl/pdf/eure/v34n102/art06.pdf

Ascencio, M. y Pino, O. (2015). La conmutación laboral femenina en la región del Biobío: un análisis exploratorio desde la perspectiva de género. Horizontes Empresariales, 14(1), 20-29. Recuperado de http:// revistas.ubiobio.cl/index.php/HHEE/article/view/2398/2100

Bergantino, A. S. y Madio, L. (2019). Intra- and inter-regional commuting: Assessing the role of wage differentials. Papers in Regional Science, 98(2) 1085-1114. Recuperado de https://rsaiconnect.onlinelibrary. wiley.com/doi/10.1111/pirs.12394

Canales, I. y Canales, M. (2016). Trabajo, territorio y movilidad cotidiana en Chile: un estudio comparativo según tipos de conmutación laboral. VII Congreso de la Asociación Latinoamericana de Población y XX Encontro Nacional de Estudos Populacionais, Foz de Iguazú, Brasil, 17 al 22 de octubre. Recuperado de http://www.abep.org.br/xxencontro/ files/paper/534-440.pdf

Ferrada, L. M., Soza-Amigo, S. y Mancilla, C. (2019). Patagonia chilena: características de la conmutación y funcionalidad del espacio geográfico. 
Revista Geográfica Venezolana, 60(2). Recuperado de http://erevistas. saber.ula.ve/index.php/regeoven/article/view/16007

Fujita, M. y Krugman, P. (2004). La nueva geografía económica: pasado, presente y futuro. Investigaciones Regionales, 4, 177-206. Recuperado de https://www.redalyc.org/pdf/289/28900409.pdf

Krzywinski, M., Schein, J., Birol, I., Connors, J., Gascoyne, R., Horsman, D., Jones, S. y Marra, M. (2009). Circos: An information aesthetic for comparative genomics. Genome Research, 19(9), 1639-1645. Recuperado de https:/www.ncbi.nlm.nih.gov/pmc/articles/PMC2752132/

Le Masne, P. (1988). Le système productiffrançais face a ses voisins européens. Troisième Colloque de Comptabilité Nationale, París, 12 a 14 de diciembre.

Lira, L. y Quiroga, B. (2008). Técnicas de análisis regional. Santiago de Chile: CEPAL (Manuales, 59). Recuperado de https://repositorio.cepal. org/handle/11362/5500

Massey, D., Arango, J., Hugo, G., Kouaouci, A., Pellegrino, A. y Taylor, J. E. (1993). Theories of international migration: A review and appraisal. Population and Development Review, 19(3), 431-466. Recuperado de https://www.jstor.org/stable/2938462?seq=1\#metadata_info_tab_ contents

Muñoz, F. (2012). Enfrentado el desajuste entre la oferta del Sistema Educacional Técnico y la demanda de capital humano en la industria minera. (Tesis de maestría en Gestión y Política Pública, Universidad de Chile). Recuperado de http://repositorio.uchile.cl/bitstream/handle/ 2250/111976/cf-munoz_fr.pdf?sequence $=1$

Parr, J. (2004). The polycentric urban region: A closer inspection. Regional Studies, 38(3), 231-240. Recuperado de https://www.tandfonline.com/ doi/abs/10.1080/003434042000211114

Parr, J. (2008). Cities and regions: Problems and potentials. Environment and Planning A: Economy and Space, 40(12), 3009-3026. Recuperado de https://journals.sagepub.com/doi/pdf/10.1068/a40217

Pino, O., Concha, G. y Fuentes, S. (2016). Análisis comparado de la conmutación interregional en Chile (2011-2014). Multidisciplinary Business Review, 9(1), 53-66. Recuperado de http://www.asfae.cl/journalmbr/ images/stories/2016Artculo06.pdf

Roberts, D. (1998). Rural-urban interdependencies: Analysis using an inter-regional SAM model. European Review of Agricultural Economics, 25(4), 506-527. Recuperado de https://academic.oup.com/erae/ article/25/4/506/505793 
Roberts, J. y Taylor, K. (2016). Intra-household commuting choices and local labour markets. Oxford Economic Papers, 69(3), 734-757. Recuperado de https://academic.oup.com/oep/article/69/3/734/2907878

Said, J. (2012). Patagonia. Buenos Aires, Argentina: Sudamericana.

Sandow, E. (2008). Commuting behaviour in sparsely populated areas: Evidence from northern Sweden. Journal of Transport Geography, 16(1), 14-27. Recuperado de https://www.sciencedirect.com/science/article/ pii/S0966692307000506

Soza-Amigo, S. (2016a). Conmutación centrípeta-centrífuga: ¿es la vocación productiva una motivación para conmutar? IDESIA, 34(1), 55-67. Recuperado de https://scielo.conicyt.cl/pdf/idesia/v34n1/art07.pdf

Soza-Amigo, S. (2016b). Impactos del turismo y de la conmutación centrípeta-centrífuga en el sur de Chile: enfoques clásicos y nuevos para su interpretación. Revista de Métodos Cuantitativos para la Economía y la Empresa, 21, 58-76. Recuperado de https://www.upo.es/revistas/ index.php/RevMetCuant/article/view/2253

Soza-Amigo, S., Mancilla, C. y Ferrada, L. (2017). El sector silvoagropecuario y su contribución al desarrollo en los principales centros nodales de la Patagonia chilena en los años 2012 y 2007. IDESIA, 35(3), 82-92. Recuperado de https://scielo.conicyt.cl/scielo.php?script=sci_arttext\& pid $=$ S0718-34292017000300081

Soza-Amigo, S., Mancilla, C. y Ferrada, L. (2018). Las especializaciones e interrelaciones productivas como factores de desarrollo en la Patagonia chilena. Revista de Geografia Norte Grande, 70, 153-181. Recuperado de https://scielo.conicyt.cl/pdf/rgeong/n70/0718-3402-rgeong-70-00153. pdf

Timmermans, B. y Boschma, R. (2014). The effect of intra- and inter-regional labour mobility on plant performance in Denmark: The significance of related labour inflows. Journal of Economic Geography, 14(2), 289-311. Recuperado de https://academic.oup.com/joeg/article/ $14 / 2 / 289 / 968187$

Webster, A. (2000). Estadística aplicada a los negocios y la economía. Santa Fe de Bogotá, Colombia: McGraw-Hill.

Yañez, M. y Acevedo, K. (2010). El mercado laboral desde una perspectiva espacial. Apuntes del CENES, 29(50), 73-97. Recuperado de https:// revistas.uptc.edu.co/index.php/cenes/article/view/46/47

Yinger, J. (1992). City and suburb: Urban models with more than one employment center. Journal of Urban Economics, 31(2), 181-205. Recuperado de https://www.sciencedirect.com/science/article/pii/009411909 $290052 \mathrm{M}$ 
Zhao, P., Lu, B. y De Roo, G. (2011). The impact of urban growth on commuting patterns in a restructuring city: Evidence from Beijing. Papers in Regional Science, 90(4), 735-755. Recuperado de https://rsaiconnect. onlinelibrary.wiley.com/doi/full/10.1111/j.1435-5957.2010.00343.x

\section{Acerca de los autores}

Claudio Mancilla es ingeniero comercial y maestro en Ciencias Sociales por la Universidad de Los Lagos; es doctor en Economía Aplicada por la Universitat Autònoma de Barcelona, España. Es académico del Departamento de Ciencias Administrativas y Económicas de la Universidad de Los Lagos, e investigador del proyecto Observatorio Laboral de la Región de Los Lagos-Chile. Sus líneas de investigación se relacionan con desarrollo local y regional, emprendimiento, y gobernanza universitaria. ORCID: https://orcid.org/0000-0003-2864-2112

Entre los trabajos en los que ha abordado estas temáticas se encuentran: Abello, J. y Mancilla, C. (2018). Análisis multiteórico de los gobiernos corporativos universitarios sobre la divulgación de información. Opción. Revista de Ciencias Humanas y Sociales, 34(86), 358-392. Recuperado de https://produccioncientificaluz.org/index.php/opcion/article/ view/23848

Soza-Amigo, S., Mancilla, C. y Ferrada, L. M. (2017). El sector silvoagropecuario y su contribución al desarrollo en los principales centros nodales de la Patagonia chilena en los años 2012 y 2007. IDESIA, 35(3), 81-92. Recuperado de https://scielo.conicyt.cl/scielo.php?script=sci_ arttext\&pid=S0718-34292017000300081

Mancilla, C. y Amorós, J. E. (2015). Entrepreneurship in regions: Differentiated impacts of the socio-cultural and gender types. Academia. Revista Latinoamericana de Administración, 28(1), 45-76. Recuperado de https://www.researchgate.net/publication/273457262_Entrepreneurs hip_in_Regions_Differentiated_Impacts_of_the_Socio_Cultural_and_ Gender_Types

Sergio Soza-Amigo es ingeniero naval, maestro en Economía y Gestión Regional por la Universidad Austral de Chile, y doctor por la Universidad de Oviedo, España. Es académico del Instituto de Gestión e Industria en la Universidad Austral de Chile, en la ciudad de Puerto Montt, y miembro del Consejo Asesor de la Vicerrectoría de Investigación, Desarrollo y Creación 
Artística de la misma universidad. Su objetivo profesional es colaborar en la formación de especialistas funcionales en el campo de la economía aplicada, para que puedan proponer y tomar decisiones acertadas en cuanto a generación, evaluación e impacto de las políticas públicas orientadas a determinado desarrollo o cambio estructural. Su especialización la ha centrado en el análisis input-output, el análisis regional, y el desarrollo local y regional. ORCID: https://orcid.org/0000-0002-8256-6782

Entre los trabajos que ha desarrollado se encuentran:

Soza-Amigo, S., Mancilla, C. y Ferrada, L. M. (2018). Las especializaciones e interrelaciones productivas como factores de desarrollo en la Patagonia chilena. Revista de Geografia Norte Grande, 70, 1-29. Recuperado de https://scielo.conicyt.cl/scielo.php?script=sci_arttext\& pid $=$ S0718-34022018000200153

Soza-Amigo, S., Aroca, P. y Rueda-Cantuche, J. M. (2018). El desarrollo y los campos de influencia. Revista de Investigaciones Regionales, 40, 7-34. Recuperado de https://investigacionesregionales.org/wp-content/ uploads/sites/3/2018/06/1.-Soza.pdf

Soza-Amigo, S. y Aroca, P. (2018). Productive structural and development:

The case of the Chilean economy. Journal of Quantitative Methods for Economics and Business Administration, 26, 175-202. Recuperado de https:/www.upo.es/revistas/index.php/RevMetCuant/article/view/2541

Luz María Ferrada es ingeniera comercial y maestra en Economía y Gestión Regional por la Universidad Austral de Chile, y doctora en Economía Aplicada y Análisis Regional por la Universidad de Valladolid, España. Es académica del Departamento de Ciencias Administrativas y Económicas de la Universidad de Los Lagos y directora del proyecto Observatorio Laboral de la Región de Los Lagos-Chile. Pertenece a la Sociedad Chilena de Estudios Regionales (Socher), donde también ha sido presidenta. Sus líneas de investigación se relacionan con economía regional, mercado laboral y recursos naturales. ORCID: https://orcid.org/0000-0002-0481-7670

Entre los trabajos que ha desarrollado se encuentran:

Ferrada, L. M., Soza-Amigo, S. y Mancilla, C. (2019). Patagonia chilena: características de la conmutación y funcionalidad del espacio geográfico. Revista Geográfica Venezolana, 60(2), 346-359. Recuperado de http://www.saber.ula.ve/handle/123456789/46557

Ferrada, L. M. y Ferrada, M. A. (2018). Employment quality of senior citizens in Chile, an active aging factor. Papeles de Población, 24(95), 4368. Recuperado de https://rppoblacion.uaemex.mx/article/view/10092 
Ferrada, L. M. (2017). Job satisfaction in Chile: Geographic determinants and differences. CEPAL Review, 123, 126-145. Recuperado de https://www. cepal.org/en/publications/43452-job-satisfaction-chile-geographicdeterminants-and-differences

Recepción: 29 de junio de 2018. Aceptación: 3 de mayo de 2019. 